\title{
Heterometallic Seed-Mediated Growth of Monodis- perse Colloidal Copper Nanorods with Widely Tunable Plasmonic Resonances
}

Soojin Jeong, Yang Liu, Yaxu Zhong, Xun Zhan, Yuda Li, Yi Wang, Phoebe M. Cha, Jun Chen, Xingchen Ye*

Department of Chemistry, Indiana University, 800 East Kirkwood Avenue, Bloomington, Indiana 47405, USA.

* Correspondence should be addressed to X.Y. (xingye@ indiana.edu). 


\section{Contents}

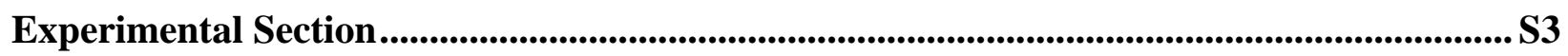

Text

HRTEM and Electron Diffraction Studies of Cu NRs ..........................................................S6

Figure S1-S2

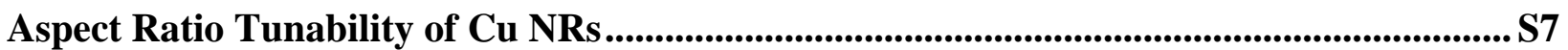

Table S1, Figure S3-S11

Finite-Difference Time-Domain (FDTD) Simulation Results ................................................S17

Table S2, Figure S12-S15

Mechanistic Studies of Cu NR Growth.......................................................................................S22

Text, Table S3-S5, Figure S16-S20

Analysis of Shape Impurities Obtained during Cu NR Formation.........................................S29

Figure S21-S25

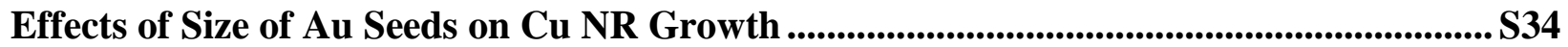

Text, Table S6-S8, Figure S26-S32

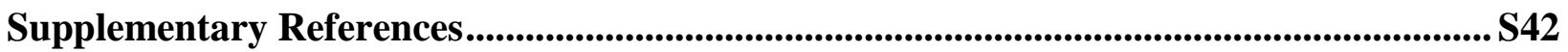




\section{Experimental Section}

Chemicals. Tetrachloroauric acid trihydrate $\left(\mathrm{HAuCl}_{4} \cdot 3 \mathrm{H}_{2} \mathrm{O}, 99 \%\right)$, borane tert-butylamine (TBAB, 97\%), copper(II) chloride dihydrate $\left(\mathrm{CuCl}_{2} \cdot 2 \mathrm{H}_{2} \mathrm{O}, 99.999 \%\right)$, oleylamine (OLAM-SA, 70\%), tetrachloroethylene (TCE, 99.5\%), acetone (99.5\%), 2-propanol (IPA, 99.5\%), styrene ( $\geq$ 99\%), ethyl $\alpha$-bromoisobutyrate (EBIB, 98\%), copper(II) bromide (99\%), tin(II) 2-ethylhexanoate $\left(\mathrm{Sn}(\mathrm{EH})_{2}\right.$, 92.5-100.0\%), tris(2-dimethylaminoethyl)amine ( $\left.\mathrm{Me}_{6} \mathrm{TREN}, 97 \%\right)$, triethylamine $\left(\mathrm{Et}_{3} \mathrm{~N}, \geq 99.5 \%\right.$ ), $N, N$-dimethylformamide (DMF, anhydrous, 99.8\%) were purchased from Sigma Aldrich. Calcium hydride (90-95\%, 2 mm \& down) were purchased from Alfa Aesar. Oleylamine (OLAM-TCI, 50\%) and 2-aminoethanethiol ( $\geq 95 \%$ ) were purchased from TCI America. Anhydrous toluene and tetrahydrofuran (THF) were obtained from a custom-built solvent purification system. OLAM-SA was used as received without further purification whereas OLAM-TCI was pre-dried at $100{ }^{\circ} \mathrm{C}$ under vacuum for four hours and stored inside a $\mathrm{N}_{2}$-filled glovebox. Styrene was washed with $10 \% \mathrm{w} / \mathrm{v}$ sodium hydroxide solution to remove inhibitor, and distilled under vacuum prior to use.

Synthesis of Au NC seeds. Au NCs were synthesized according to previously reported methods with minor modifications. ${ }^{1,2}$ All syntheses were carried out under nitrogen atmosphere using standard Schlenk line techniques. In a typical synthesis of $9 \mathrm{~nm}$ Au NCs, $10 \mathrm{~mL}$ of pre-dried OLAM-TCI was loaded into a $100 \mathrm{~mL}$ three-neck flask. After degassing under vacuum for $30 \mathrm{~min}$ at $25^{\circ} \mathrm{C}, 10 \mathrm{~mL}$ of anhydrous toluene was injected into the flask followed by flushing with $\mathrm{N}_{2}$ for $10 \mathrm{~min}$. Afterward, $0.25 \mathrm{mmol}(98 \mathrm{mg})$ of $\mathrm{HAuCl}_{4} \cdot 3 \mathrm{H}_{2} \mathrm{O}$ was added into the mixture followed by purging with $\mathrm{N}_{2}$ for another $10 \mathrm{~min}$. Subsequently, a solution mixture of $0.25 \mathrm{mmol}(22 \mathrm{mg})$ of TBAB, $1 \mathrm{~mL}$ of OLAM-TCI, and $1 \mathrm{~mL}$ of anhydrous toluene was swiftly injected into the reaction mixture, which was kept under stirring for another hour at $15{ }^{\circ} \mathrm{C}$. Au NCs were isolated via precipitation with $60 \mathrm{~mL}$ of acetone followed by centrifugation at $6000 \mathrm{rpm}$ for $5 \mathrm{~min}$. The precipitates were re-dispersed in anhydrous toluene. Au NCs of different sizes were synthesized by varying the amount of TBAB or reaction temperature, as detailed in Table S6.

Seeded growth of $\mathbf{C u}$ NRs. In a typical reaction, $0.5 \mathrm{mmol}(85 \mathrm{mg})$ of $\mathrm{CuCl}_{2} \cdot 2 \mathrm{H}_{2} \mathrm{O}$ was mixed with OLAM-SA in a $50 \mathrm{~mL}$ three-neck flask. The mixture was degassed under vacuum at room temperature and flushed with $\mathrm{N}_{2}$ gas for three times to remove oxygen. The mixture was then heated at $80{ }^{\circ} \mathrm{C}$ for $30 \mathrm{~min}$ to fully dissolve $\mathrm{CuCl}_{2}$ forming a blue-colored $\mathrm{Cu}$ (II)-OLAM complex. ${ }^{3}$ Subsequently, the solution temperature was raised to $180{ }^{\circ} \mathrm{C}$ when reduction of $\mathrm{Cu}$ (II) to $\mathrm{Cu}(\mathrm{I})$ occurred resulting in a yellow-colored solution. ${ }^{4}$ Afterward, a certain amount of Au NC solution (Table S1) was injected into the mixture and the reaction was allowed to proceed at $180{ }^{\circ} \mathrm{C}$ for another hour. After cooling down to room temperature, NC products were isolated by using isopropanol precipitation followed by centrifugation at $3000 \mathrm{rpm}$ for $3 \mathrm{~min}$. The precipitates were redispersed in anhydrous toluene and stored inside a $\mathrm{N}_{2}$-filled glovebox. 
Synthesis of thiol-terminated polystyrene (PS-SH). In the first step, bromine-terminated polystyrene (PS-Br) was synthesized by activators regenerated by electron transfer for atom transfer radical polymerization (ARGET-ATRP). ${ }^{5}$ Styrene $(25 \mathrm{~mL}, 218 \mathrm{mmol})$, EBIB $(640 \mu \mathrm{L}, 4.36$ $\mathrm{mmol}), \mathrm{CuBr}_{2}(10 \mathrm{mg}, 40 \mu \mathrm{mol}), \mathrm{Me}_{6} \mathrm{TREN}(116 \mu \mathrm{L}, 440 \mu \mathrm{mol})$ and anhydrous toluene $(12.5 \mathrm{~mL})$ were added into a $100 \mathrm{~mL}$ round-bottom flask. After purging with $\mathrm{N}_{2}$ for $30 \mathrm{~min}, \mathrm{Sn}(\mathrm{EH})_{2}(140$ $\mu \mathrm{L}, 440 \mu \mathrm{mol}$ ) was injected via a microsyringe. The reaction mixture was stirred at $90{ }^{\circ} \mathrm{C}$ for 32 h. Afterward, the reaction was quenched by exposure to air and cooling in an ice bath. PS-Br was precipitated with excess methanol, vacuum filtered and re-dissolved in THF. PS-Br was again precipitated into methanol, vacuum filtered and dried under vacuum at $40{ }^{\circ} \mathrm{C}$ overnight. In the second step, $\mathrm{PS}-\mathrm{Br}$ was converted to PS-SH using previously reported method with slight modification. ${ }^{6}$ PS-Br $(5.5 \mathrm{~g}, 1 \mathrm{mmol})$ and triethylamine $(5.7 \mathrm{~mL}, 41 \mathrm{mmol})$ were dissolved in $55 \mathrm{~mL}$ of DMF in a $100 \mathrm{~mL}$ round bottom flask. Afterward, 2-aminoethanethiol (1.18 g, $15 \mathrm{mmol})$ was injected and the solution was purged with $\mathrm{N}_{2}$ for another $30 \mathrm{~min}$. The reaction mixture was stirred at room temperature for $64 \mathrm{~h}$. PS-SH was precipitated with excess methanol, vacuum filtered and then dried under vacuum at room temperature overnight.

Polymer functionalization and self-assembly of Cu NRs. In a typical process of grafting PS-SH onto $\mathrm{Cu}$ NRs, $20 \mathrm{mg}$ of 5.6k PS-SH was first dissolved in $1.6 \mathrm{~mL}$ of anhydrous THF inside a $\mathrm{N}_{2}$ filled glovebox, to which $0.5 \mathrm{~mL}$ of $\mathrm{Cu}$ NR toluene solution $(5 \mathrm{mg} / \mathrm{mL})$ was injected. Following brief sonication, the resulting mixture was kept undisturbed inside the glovebox for 12 hours. PSgrafted $\mathrm{Cu}$ NRs were purified via precipitation with anhydrous hexane followed by centrifugation at $3000 \mathrm{rpm}$ for 3 minutes. Self-assembly of $\mathrm{Cu}$ NRs were conducted by drying NR solutions on top of an immiscible ethylene glycol (EG) subphase. Typically, $15 \mu \mathrm{L}$ of $\mathrm{Cu}$ NR solution (10 $\mathrm{mg} / \mathrm{mL})$ was drop-casted onto the surface of EG inside a Teflon well $\left(1.5 \times 1.5 \times 1.5 \mathrm{~cm}^{3}\right)$. The Teflon well was covered with a microscope slide to slow down solvent evaporation. After two hours, the floating film was transferred onto a TEM grid, which was further dried inside a vacuum oven to remove residual EG.

Characterization. Low-magnification TEM images were acquired on a JEOL JEM 1400 plus microscope equipped with a $\mathrm{LaB}_{6}$ filament operating at $120 \mathrm{kV}$. TEM samples were prepared by drop-casting $\sim 10 \mu \mathrm{L}$ of NC solution onto 300-mesh carbon-coated copper grids (Ted Pella). Highresolution TEM (HRTEM) imaging, coherent nano-area electron diffraction (CNED), nanobeam electron diffraction (NBED) and scanning TEM energy-dispersive X-ray spectroscopy (STEMEDS) analysis were performed on a 300-kV JEOL JEM 3200FS TEM equipped with a Gatan 4k $\times 4 \mathrm{k}$ Ultrascan 4000 camera. SEM-EDS analysis was carried out over a sample area of at least 10 $\mu \mathrm{m}^{2}$ on a Zeiss Auriga 60 FIB-SEM equipped with the X-Max $50 \mathrm{~mm}^{2}$ silicon drift detector and the AZtec software package (Oxford Instrument). Samples for SEM-EDS analysis were deposited onto 300-mesh nickel TEM grids. Powder XRD patterns were collected on a PANalytical Empyrean X-ray diffractometer operated at $40 \mathrm{kV}$ and $45 \mathrm{~mA}$. Samples for XRD were prepared by dropcasting NC solutions onto single-crystalline Si substrates. UV-Vis-NIR extinction spectra were 
acquired on a Varian Cary 5000 UV-Vis-NIR spectrophotometer (Agilent). Samples for UV-VisNIR measurements were dispersed in tetrachloroethylene (TCE). ${ }^{1} \mathrm{H}$ NMR spectra were recorded on a Varian Inova $600 \mathrm{MHz}$ spectrometer at $298 \mathrm{~K}$ using $\mathrm{CDCl}_{3}$ as solvent. Gel permeation chromatography (GPC) was conducted on a Waters Breeze GPC system equipped with a Waters 1525 binary HPLC pump, a 1500 column heater and a 2414 refractive index detector. THF was used as the eluent with a flow rate of $1 \mathrm{~mL} / \mathrm{min}$. The molecular weight distribution of polymers was calculated based on calibrations with commercial polystyrene standards. Inductively coupled plasma optical emission spectrometry (ICP-OES) measurement were done on an Agilent 730 series ICPOES spectrometer. For each measurement, NCs were digested with $3 \mathrm{~mL}$ of aqua regia (a mixture of $\mathrm{HCl}$ and $\mathrm{HNO}_{3}$ in 3:1 volume ratio) and the resulting solution was further diluted 50 times with water before testing.

Finite-difference time-domain (FDTD) simulations. Extinction spectra of $\mathrm{Cu}$ NRs were calculated by using FDTD simulation software package FDTD Solutions 8.21 developed by Lumerical Solutions Inc. ${ }^{7-10}$ Dielectric functions from default materials database of FDTD Solutions were used for simulations. Individual $\mathrm{Cu} \mathrm{NR}$ was modelled as a core-shell spherocylinder with a $\mathrm{Cu} \mathrm{NR}$ core and a thin, conformal $\mathrm{Cu}_{2} \mathrm{O}$ shell $(<2 \mathrm{~nm})$. The refractive index of surrounding medium was set to be 1.506 considering that $\mathrm{Cu}$ NRs were dispersed in tetrachloroethylene (TCE) for UV-VisNIR measurements. ${ }^{11} \mathrm{~A}$ total field scattered field source (wavelength range: 400-3000 nm) was used to simulate the absorption and scattering properties of $\mathrm{Cu}$ NRs. A global non-uniform threedimensional meshing was employed for the whole simulation domain with additional uniform fine mesh $(0.1 \mathrm{~nm} \times 0.05 \mathrm{~nm} \times 0.05 \mathrm{~nm})$ applied inside the scattered-field region. The polarization direction was positioned parallel to the long-axis of NR, while the direction of propagation was set to be orthogonal to the NR's long-axis. Frequency-domain field and power monitors were used to obtain the electrical field profile of NRs. Near-field enhancement (NFE) maps were simulated at the wavelength of maximum extinction. 


\section{HRTEM and Electron Diffraction Studies of $\mathrm{Cu}$ NRs}

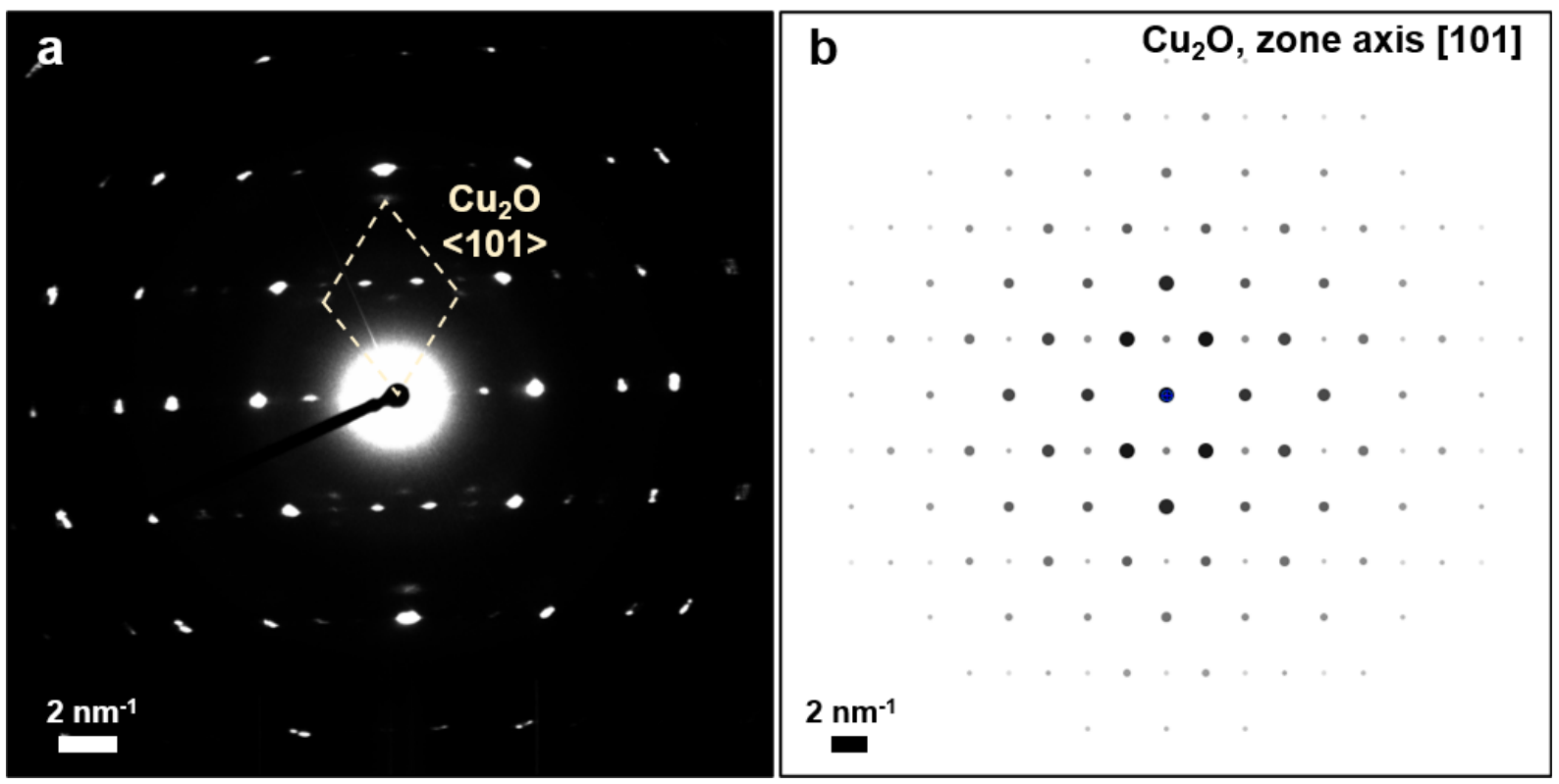

Figure S1. (a) Experimental SAED pattern of $\mathrm{Cu}$ NRs with additional diffraction spots indexed to the cubic $\mathrm{Cu}_{2} \mathrm{O}$ phase (space group $\mathrm{Pn} \overline{3} \mathrm{~m}$ ) produced by surface oxidation. (b) Simulated SAED pattern of the cubic $\mathrm{Cu}_{2} \mathrm{O}$ phase along the [101] zone axis.
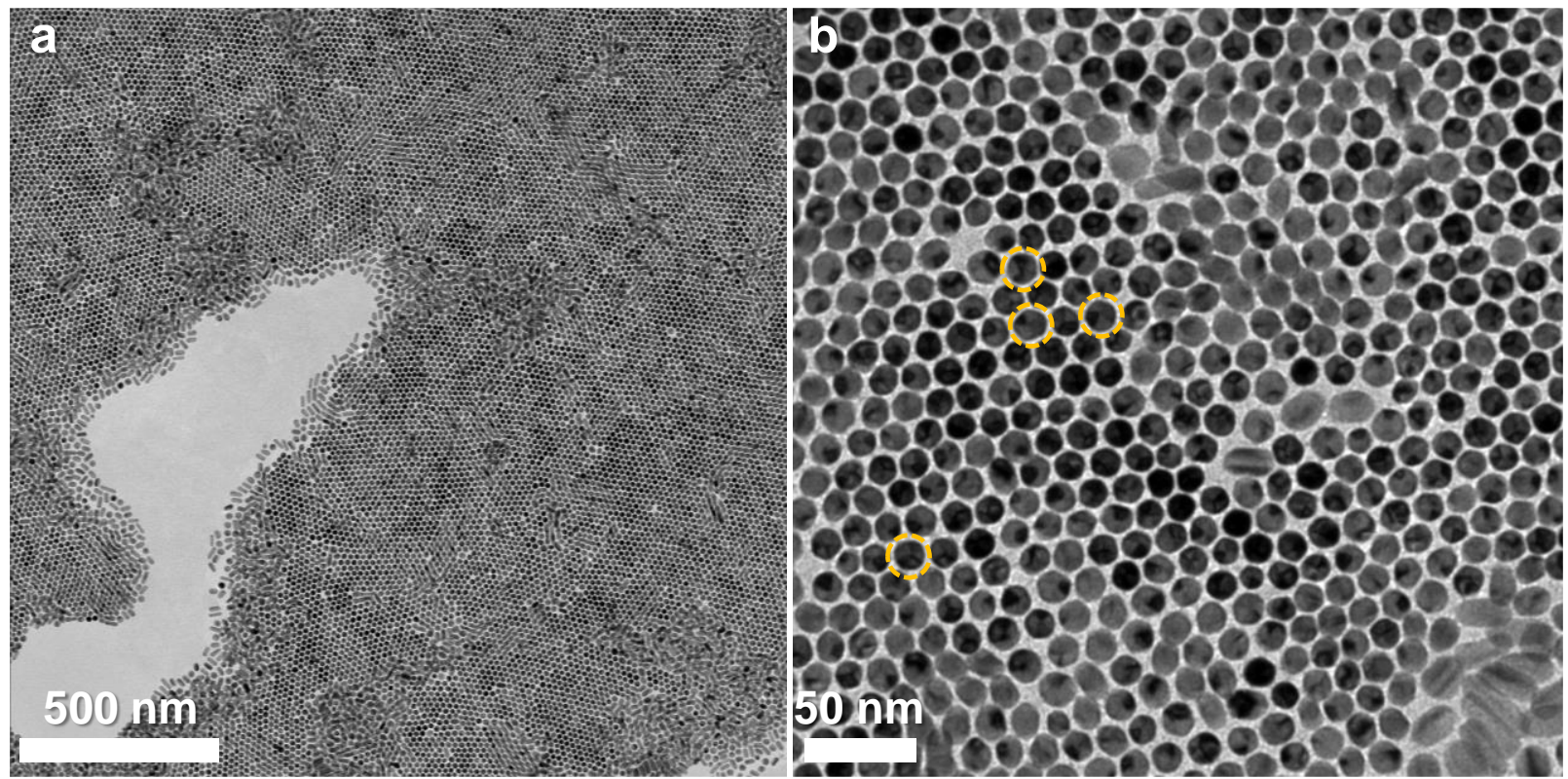

Figure S2. (a) Low- and (b) high-magnification TEM images of vertically aligned Cu NRs (average AR: 2.8). The pentagonal cross-section of multi-twinned NRs can be readily resolved (see examples highlighted with dashed yellow circles). 


\section{Aspect ratio tunability of $\mathrm{Cu}$ NRs}

Table S1. Summary of aspect ratio tunability of $\mathrm{Cu}$ NRs by varying the amount of Au seeds.

\begin{tabular}{cccc}
\hline $\begin{array}{c}\text { Diameter of Au NCs } \\
(\mathbf{n m})\end{array}$ & $\begin{array}{c}\text { Optical density } \\
(\text { OD) of Au } \\
\text { seed solution }\end{array}$ & $\begin{array}{c}\text { Injection volume of Au } \\
\text { seed solution }(\mathbf{m L})\end{array}$ & $\begin{array}{c}\text { Aspect ratio } \\
\text { of Cu NRs }\end{array}$ \\
\hline \multirow{2}{*}{0.23} & 0.75 & $2.8 \pm 0.6$ \\
& 0.50 & $5.1 \pm 1.4$ \\
& 0.35 & $8.0 \pm 1.8$ \\
& 0.26 & $9.0 \pm 2.1$ \\
& 0.17 & $13.1 \pm 4.5$ \\
\hline
\end{tabular}




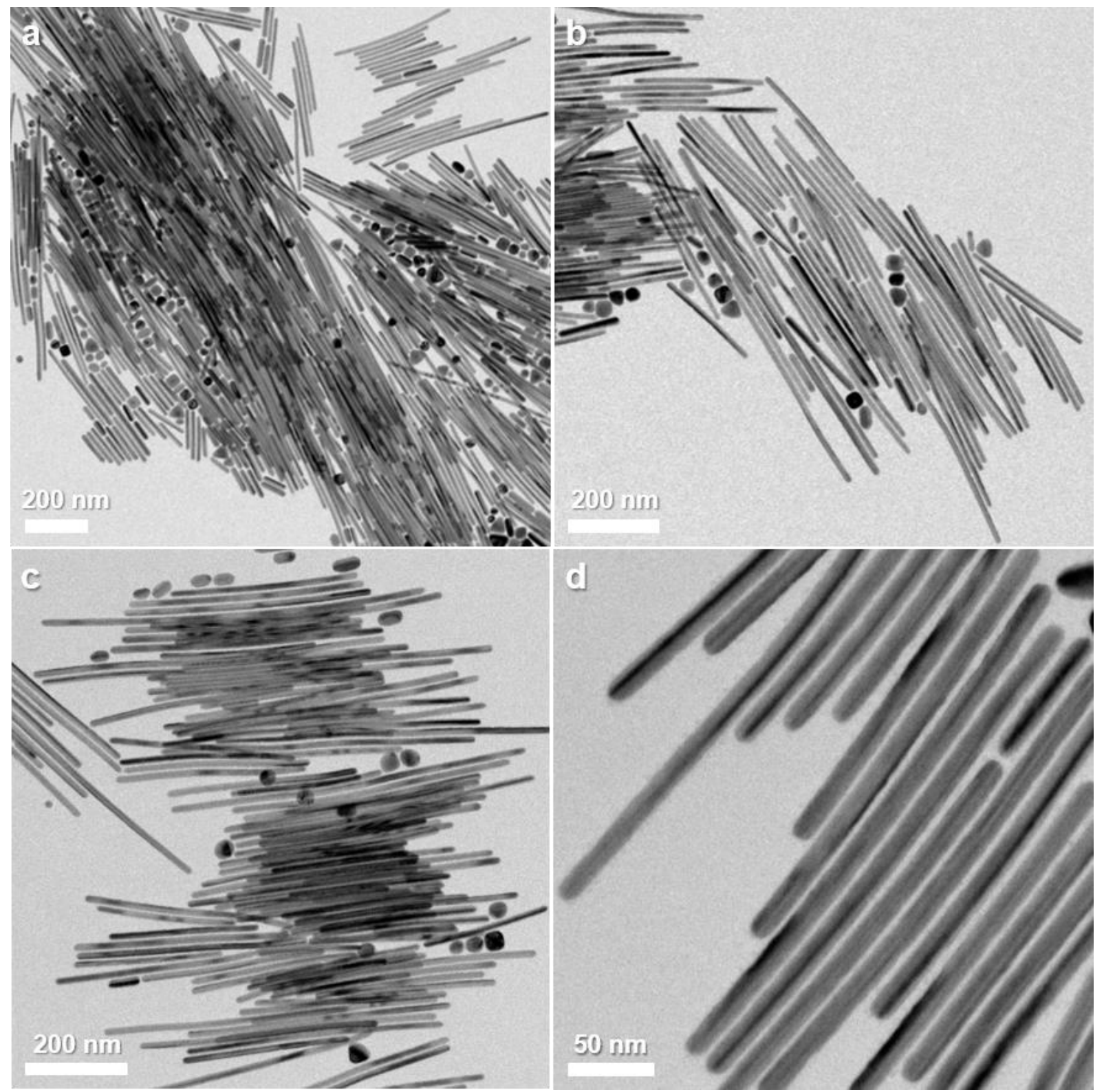

Figure S3. TEM images of ultra-long Cu NRs (average AR: 34.5) synthesized by using $0.10 \mathrm{~mL}$ of $9 \mathrm{~nm} \mathrm{Au}$ seed solutions (OD =0.23). A fraction of NRs was observed to precipitate during the course of reaction, which might have contributed to increased sample polydispersity. 

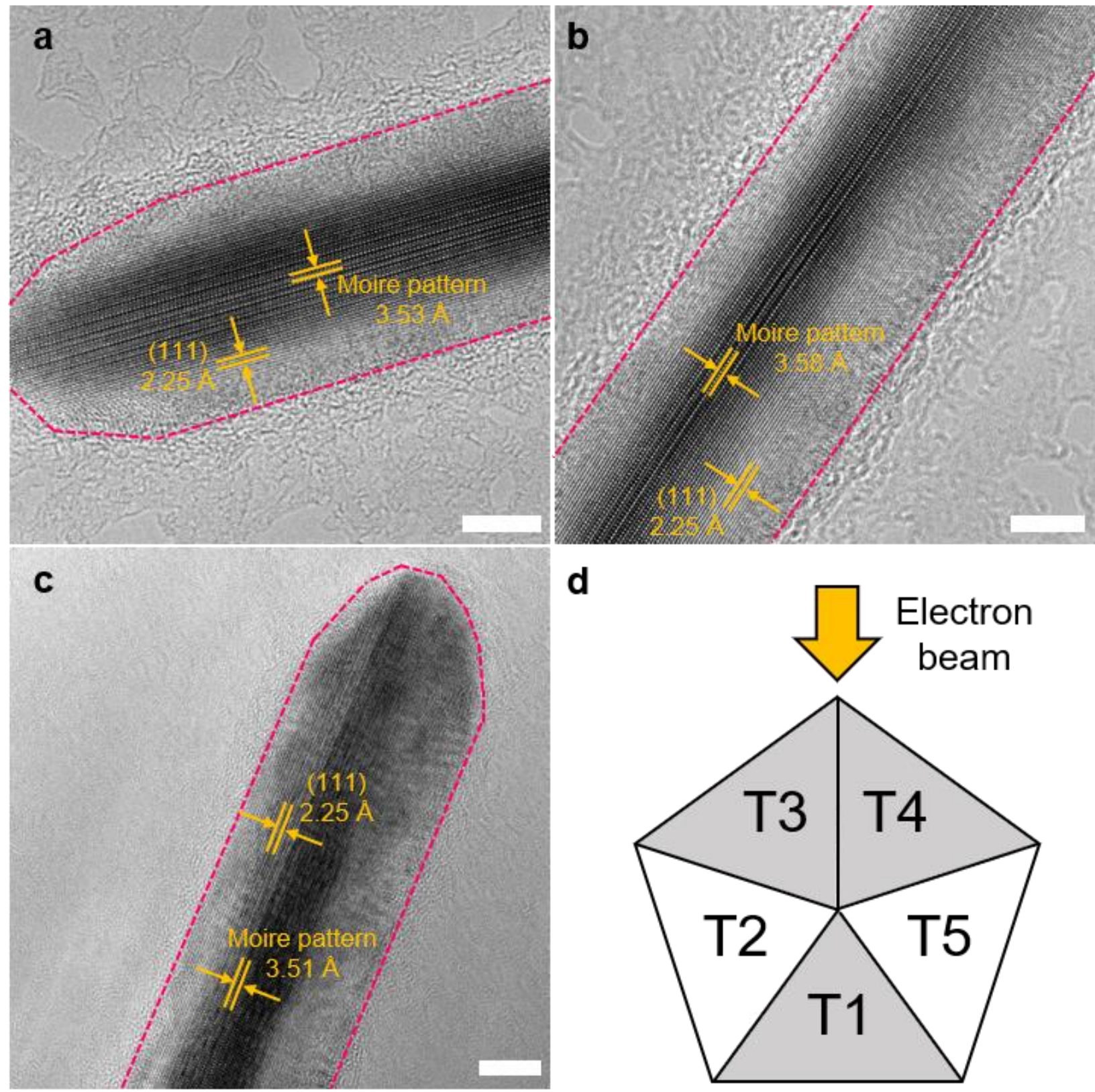

Figure S4. (a-c) HRTEM images of $\mathrm{Cu}$ NRs with the AR of (a) 3.8, (b) 6.5, and (c) 10.7. Both (111) lattice fringes and Moiré pattern were observed, which arises from $\langle 112\rangle$ and $\langle 100\rangle$ zone combination of face-centered cubic (fcc) copper. (d) Scheme illustrating the cross-sectional view of a penta-twinned $\mathrm{Cu}$ NR showing the orientation of five domains T1 to T5 with respect to the electron beam. Scale bars: $5 \mathrm{~nm}$. 


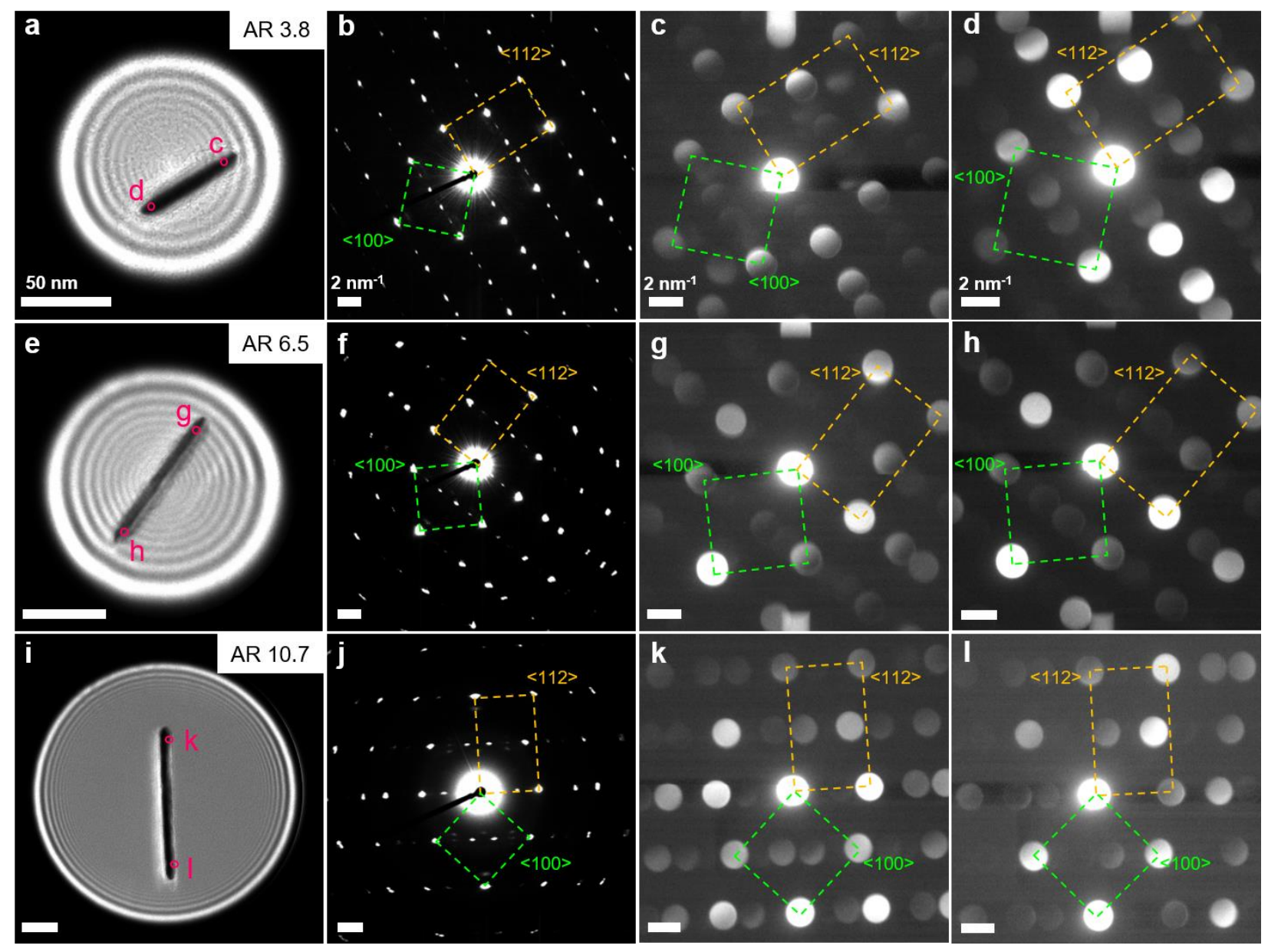

Figure S5. Electron diffraction studies of individual $\mathrm{Cu}$ NRs with the AR of (a-d) 3.8, (e-h) 6.5 and (i-l) 10.7. (b,f,j) CNED and (c,d,g,h,k,l) NBED patterns acquired from the corresponding NR shown in $(\mathrm{a}, \mathrm{e}, \mathrm{i})$. CNED patterns were recorded from the circular area defined by the size of the coherent beam (condenser aperture) covering the entire NR. NBED patterns were recorded from the spots (indicated as pink-colored circles) near the ends of individual NRs. All electron diffraction patterns showed the <112> and <100> zone combination of fcc copper, in agreement with lattice fringes seen from HRTEM images. Scale bars: $50 \mathrm{~nm}$ (TEM images) and $2 \mathrm{~nm}^{-1}$ (diffraction patterns). 


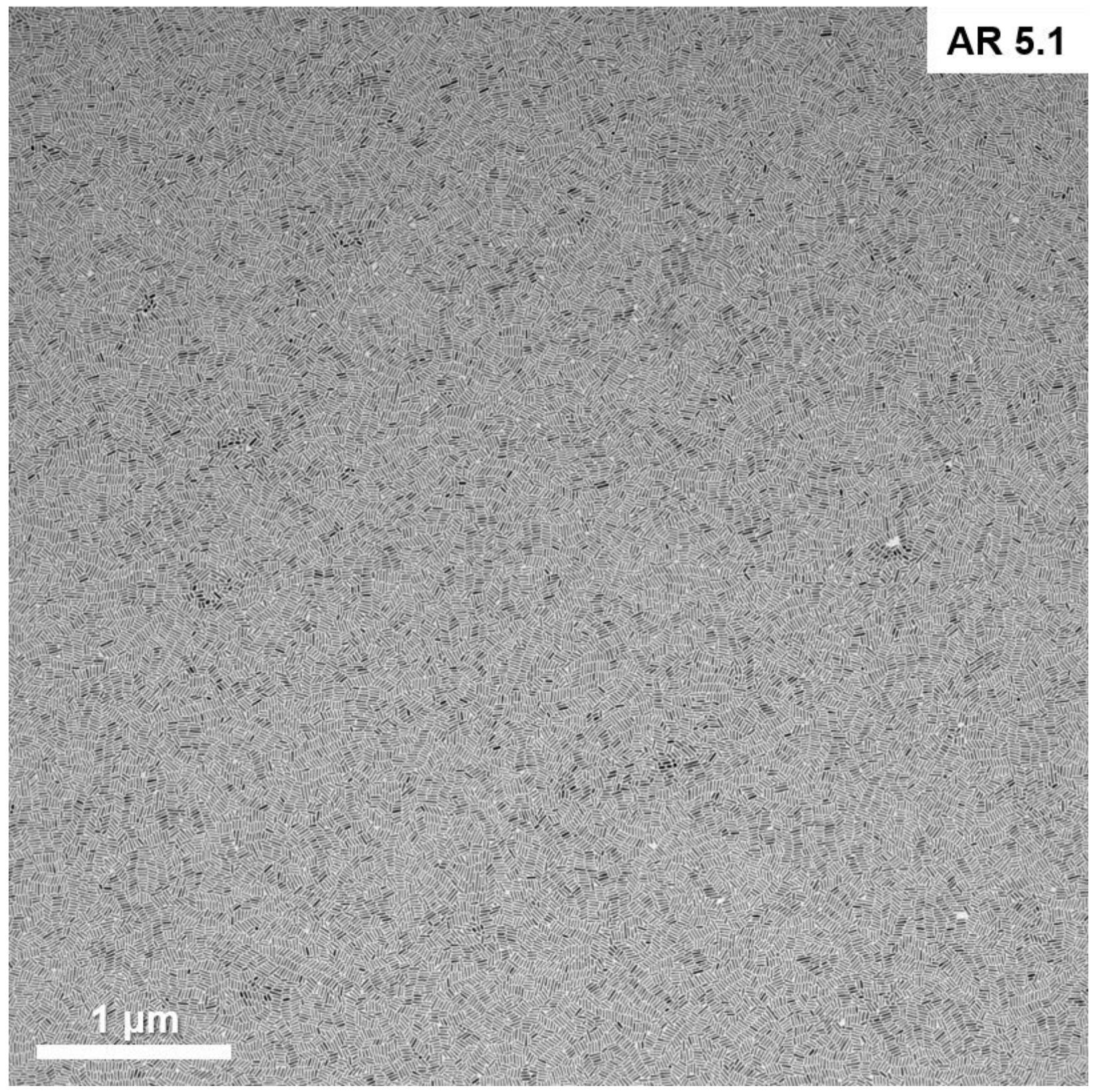

Figure S6. Low-magnification TEM image of Cu NRs (average AR: 5.1). 


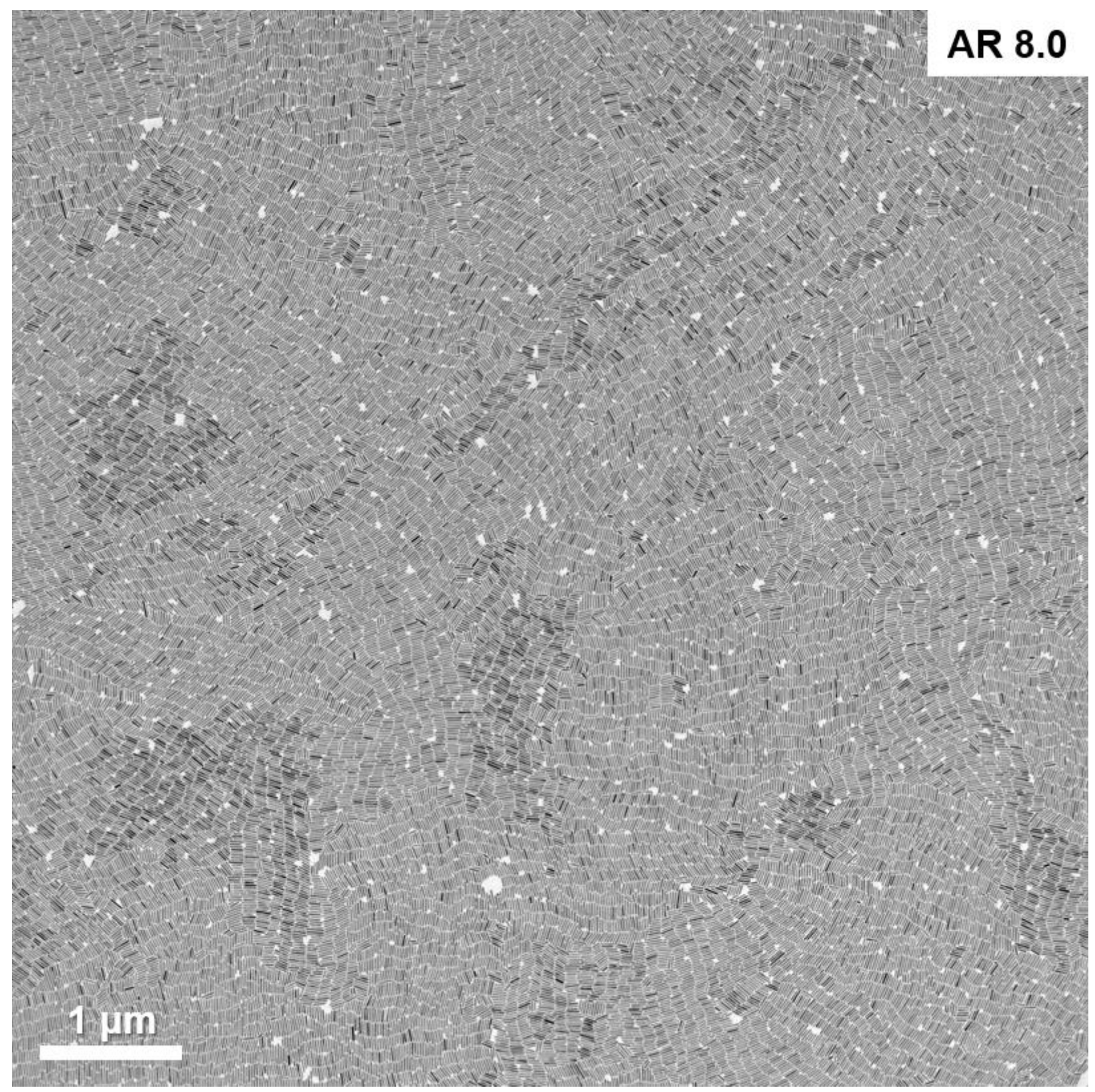

Figure S7. Low-magnification TEM image of Cu NRs (average AR: 8.0). Smectic liquid-crystalline assembly was observed for NRs with ARs exceeding 10, which can be rationalized according to the Onsager theory of hard rods (Figure S11). ${ }^{12}$ 


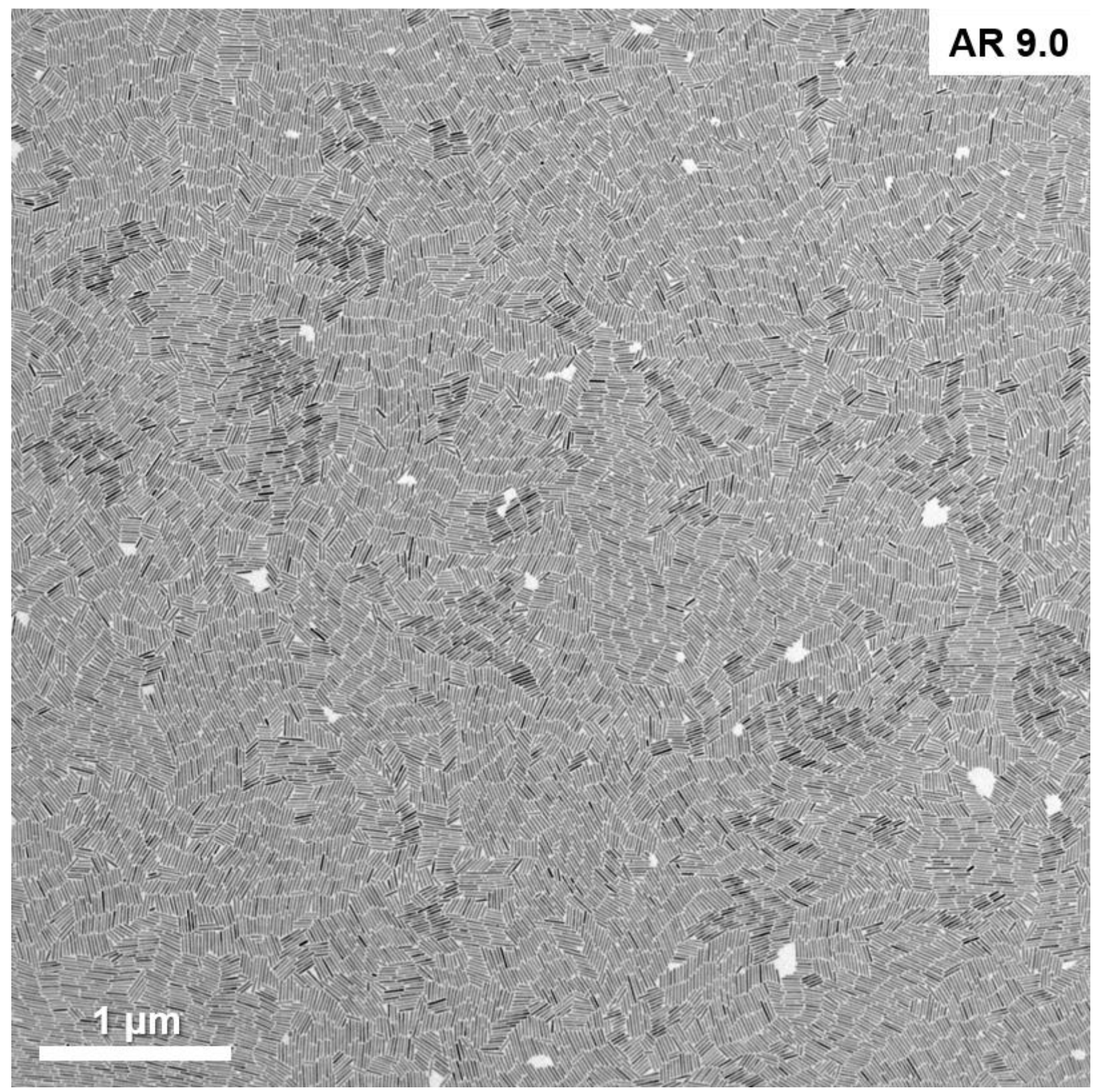

Figure S8. Low-magnification TEM image of Cu NRs (average AR: 9.0). 


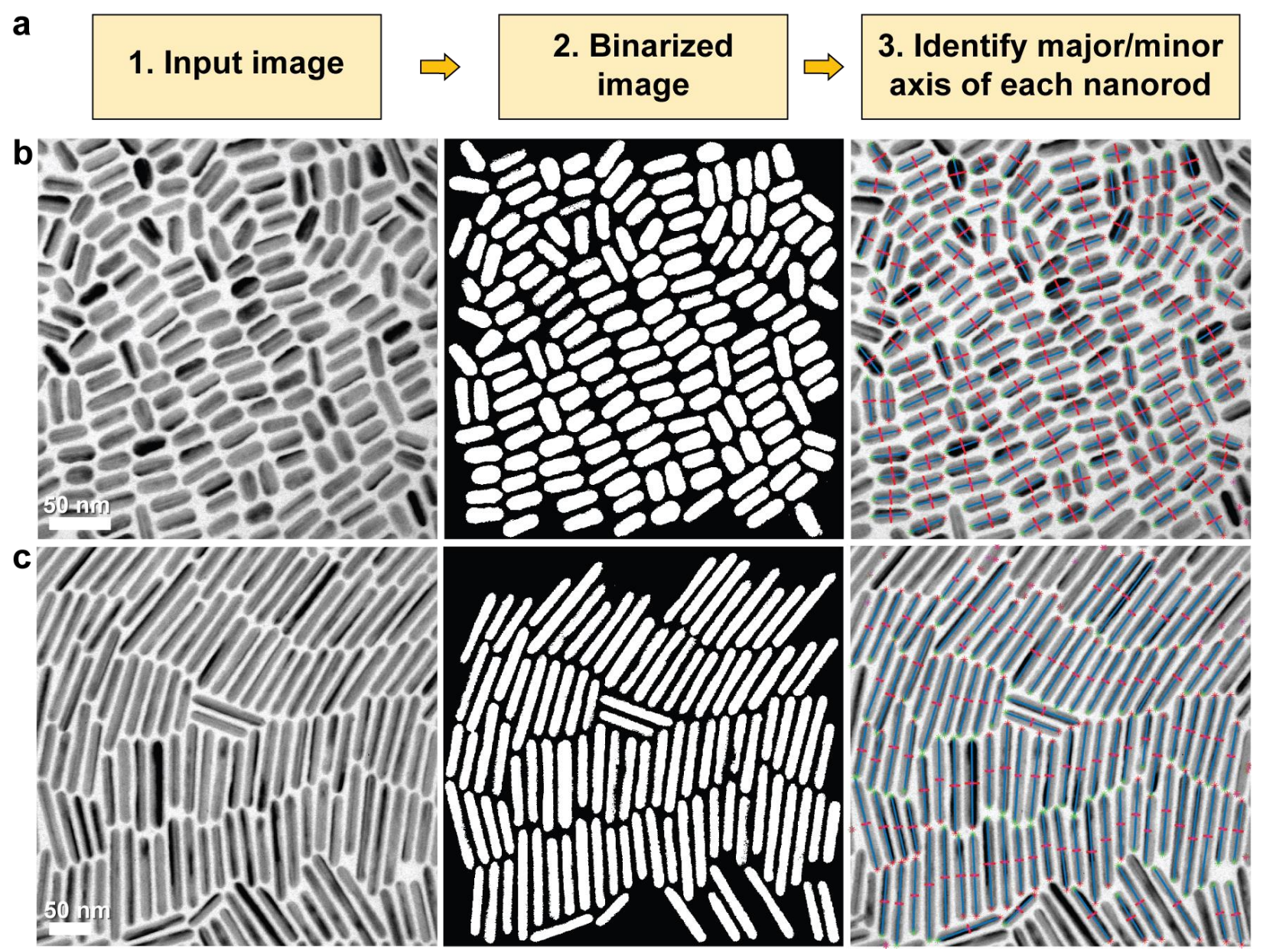

Figure S9. (a) A flowchart illustrating TEM image processing and statistical analysis of dimensions and aspect ratios of $\mathrm{Cu}$ NRs. The aspect ratio of individual NRs was determined as the ratio between the major and minor axis lengths. (b, c) Examples of TEM image analysis shown for $\mathrm{Cu}$ NRs with the average AR of (b) 2.8 and (c) 5.1. 

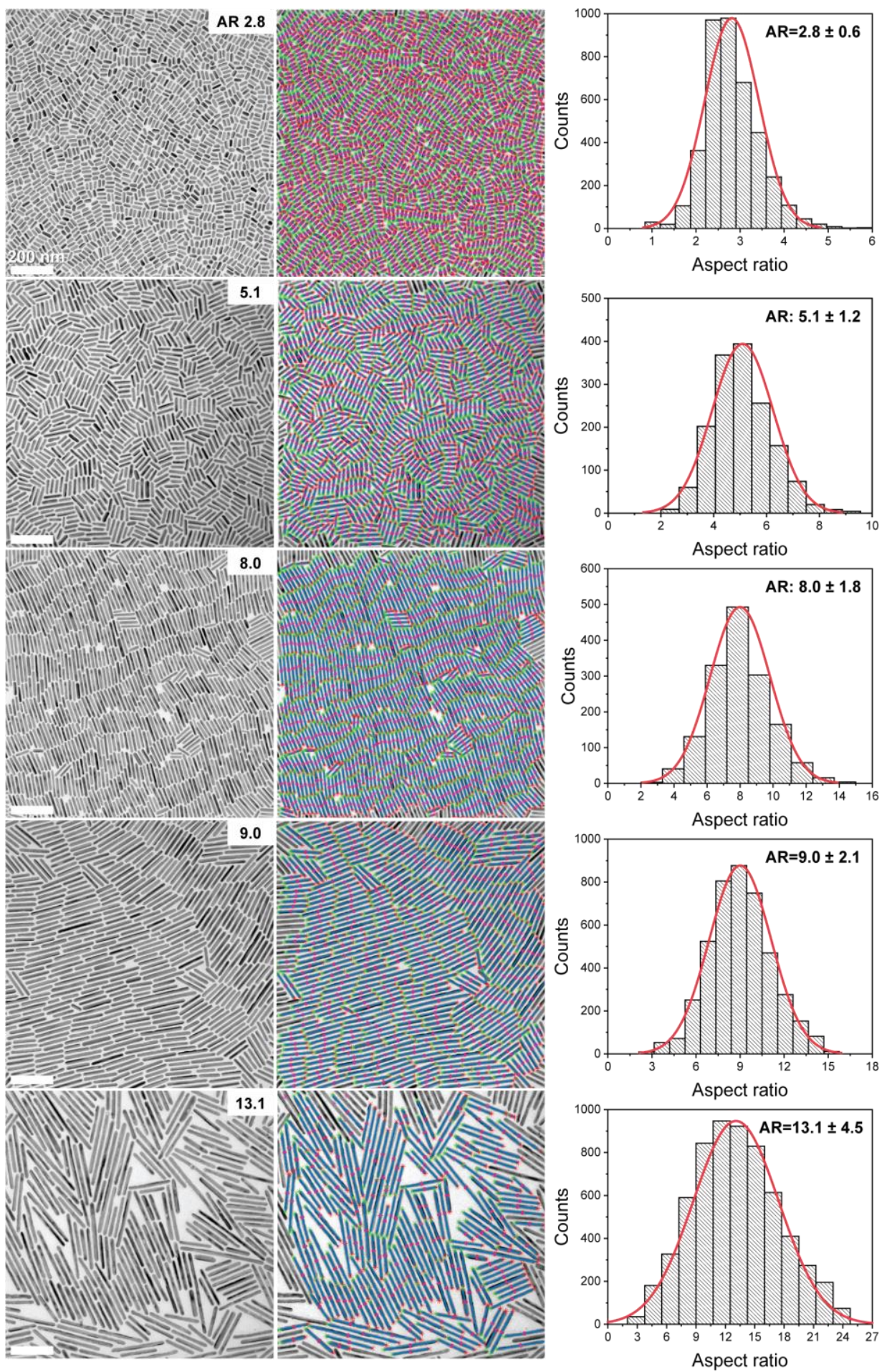

Figure S10. Statistical analysis of dimensions of $\mathrm{Cu}$ NRs with the average AR of 2.8, 5.1, 8.0, 9.0, and 13.1. Representative raw TEM images (left column), major and minor axes identified from NR tracking overlaid on the original TEM image (middle column), and histograms of NR aspect ratio (right column). The red curve represents Gaussian fit to the corresponding distribution histogram. Scale bars: $200 \mathrm{~nm}$. 

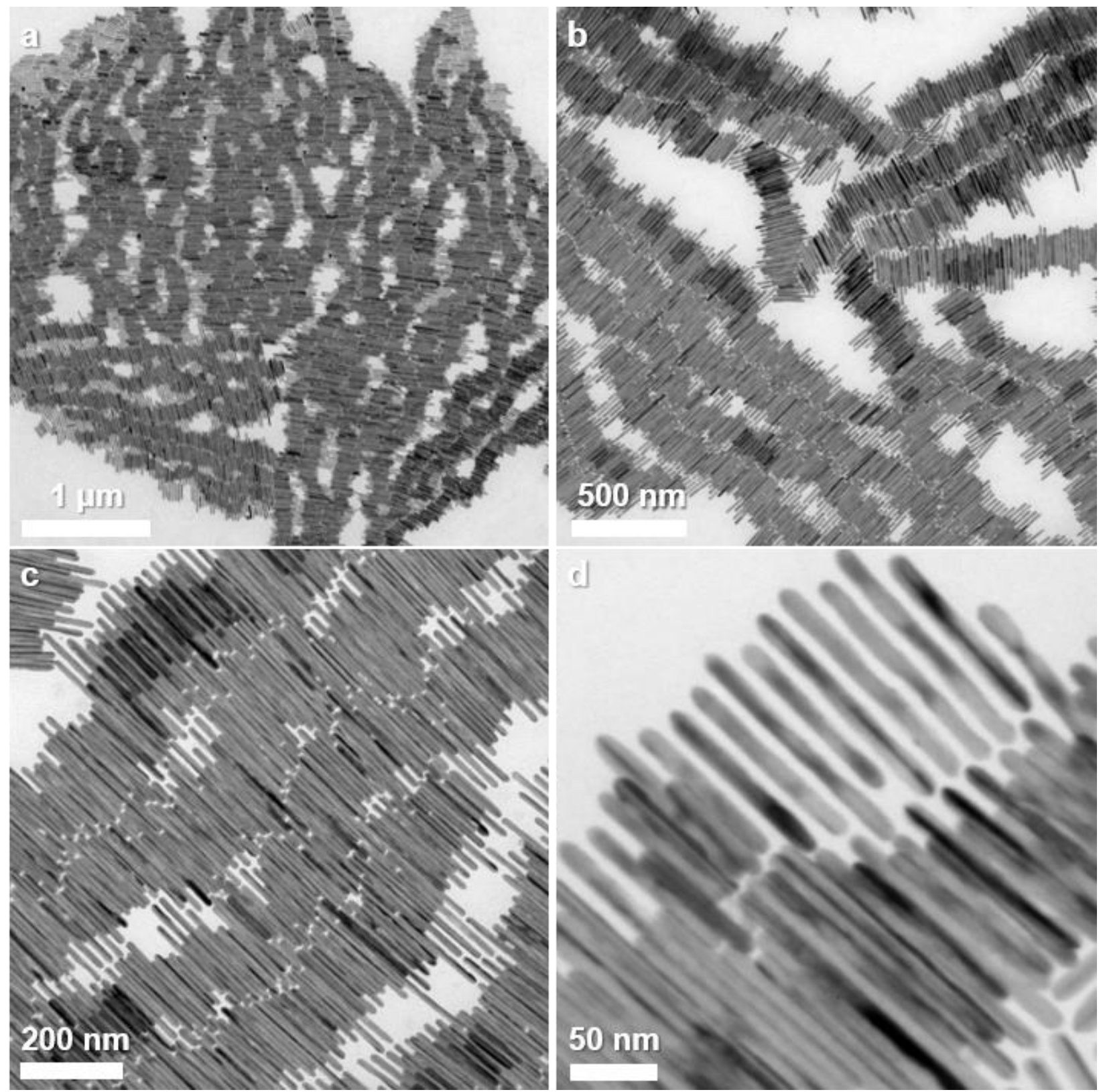

Figure S11. TEM images of liquid-crystalline assemblies of Cu NR (average AR: 13.1). Smectic liquid-crystalline assembly was observed for $\mathrm{Cu}$ NRs with ARs exceeding 10, which can be rationalized according to the Onsager theory of hard rods. ${ }^{12}$ 
Finite-Difference Time-Domain (FDTD) simulation results
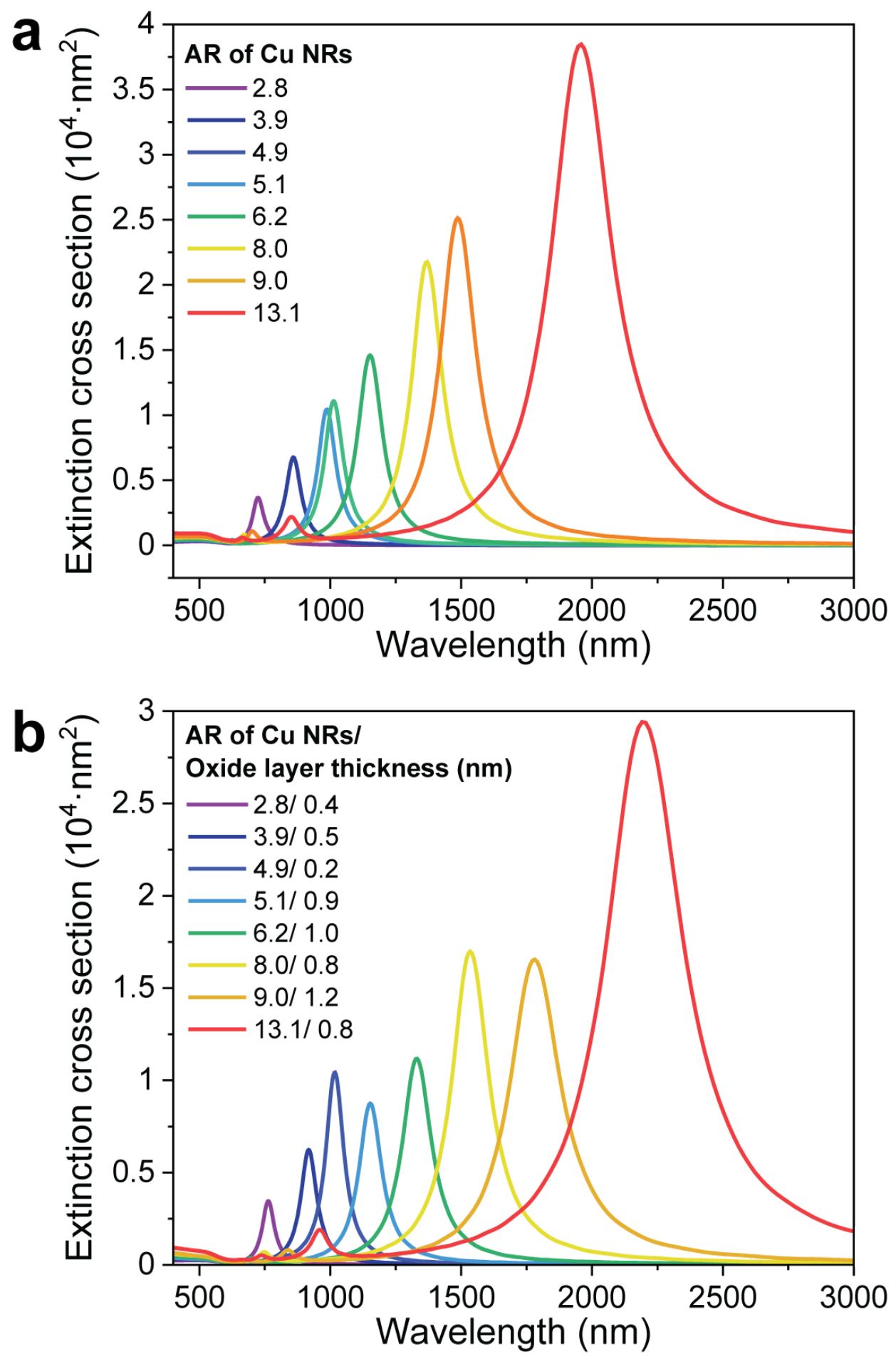

Figure S12. FDTD simulated extinction spectra of (a) pure Cu NRs and (b) Cu NRs covered with a thin surface oxide layer. The direction of electric polarization was set to be parallel to the longaxis of NR. The NR aspect ratio and surface oxide thickness are provided in the legend. 

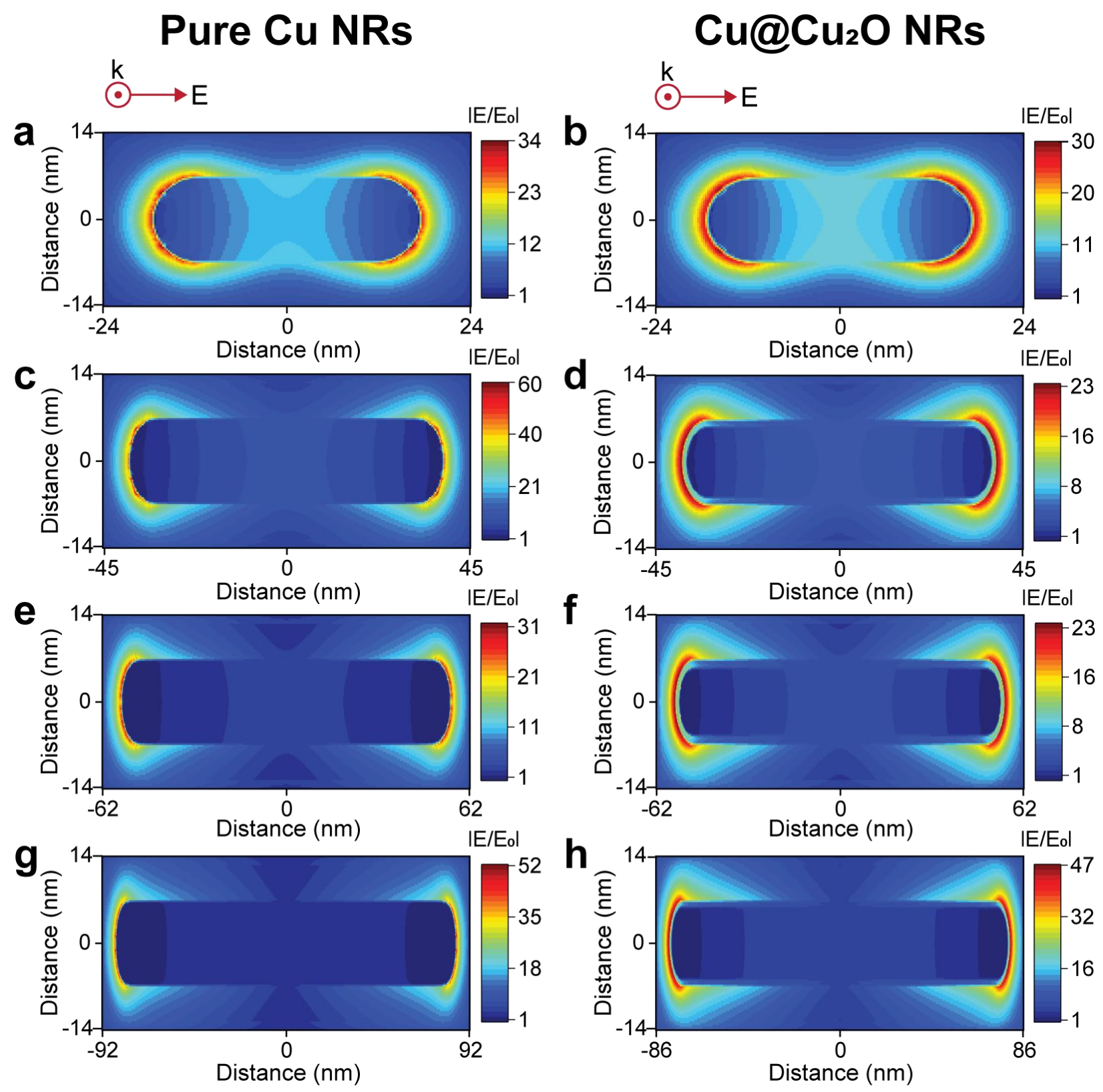

Figure S13. Near-field enhancement (NFE) maps of (a,c,e,g) pure Cu NRs and (b,d,f,h) Cu NRs with a thin surface oxide layer with the AR of (a,b) 2.8, (c,d) 6.2, (e,f) 9.0, and (g,h) 13.1. NFE maps were calculated for the extinction peak wavelength of (a) $724 \mathrm{~nm}$, (b) $763 \mathrm{~nm}$, (c) $1152 \mathrm{~nm}$, (d) $1330 \mathrm{~nm}$, (e) $1487 \mathrm{~nm}$, (f) $1781 \mathrm{~nm}$, (g) $1959 \mathrm{~nm}$ and (h) $2198 \mathrm{~nm}$. 
Table S2. Summary of FDTD simulation results of pure $\mathrm{Cu}$ NRs and $\mathrm{Cu} @ \mathrm{Cu}_{2} \mathrm{O}$ NRs. The difference between simulated and experimental maximum extinction wavelengths $\left(\lambda_{\text {sim }}-\lambda_{\text {exp }}\right)$ is shown for both $\mathrm{Cu}$ and $\mathrm{Cu} @ \mathrm{Cu}_{2} \mathrm{O}$ NRs. The thickness of $\mathrm{Cu}_{2} \mathrm{O}$ layer is varied between 0.2 and $1.5 \mathrm{~nm}$ until the closest match between $\lambda_{\text {sim }}$ and $\lambda_{\exp }$ is attained.

\begin{tabular}{|c|c|c|c|c|c|c|c|c|c|}
\hline & & \multicolumn{8}{|c|}{ Aspect ratio of NRs } \\
\hline & & 2.8 & 3.9 & 4.9 & 5.1 & 6.2 & 8.0 & 9.0 & 13.1 \\
\hline \multicolumn{2}{|c|}{$\lambda_{\exp }(\mathrm{nm})$} & 762 & 911 & 1014 & 1158 & 1333 & 1545 & 1773 & 2201 \\
\hline \multirow{2}{*}{$\begin{array}{c}\text { Pure } \\
\text { Cu NRs }\end{array}$} & $\lambda_{\text {sim }}(\mathrm{nm})$ & 724 & 859 & 985 & 1012 & 1152 & 1370 & 1487 & 1959 \\
\hline & $\lambda_{\text {sim }}-\lambda_{\exp }(\mathrm{nm})$ & -38 & -52 & -29 & -146 & -181 & -175 & -286 & -242 \\
\hline \multirow{3}{*}{$\begin{array}{c}\mathrm{Cu} @ \\
\mathrm{Cu}_{2} \mathrm{O} \\
\mathrm{NRs}\end{array}$} & $\begin{array}{l}\text { Thickness of } \\
\mathrm{Cu}_{2} \mathrm{O}(\mathrm{nm})\end{array}$ & 0.4 & 0.5 & 0.2 & 0.9 & 1.0 & 0.8 & 1.2 & 0.8 \\
\hline & $\lambda_{\text {sim }}(\mathrm{nm})$ & 763 & 917 & 1017 & 1153 & 1330 & 1535 & 1781 & 2198 \\
\hline & $\lambda_{\text {sim }}-\lambda_{\exp }(\mathbf{n m})$ & 1 & 6 & 3 & -5 & -3 & -10 & 8 & -3 \\
\hline
\end{tabular}



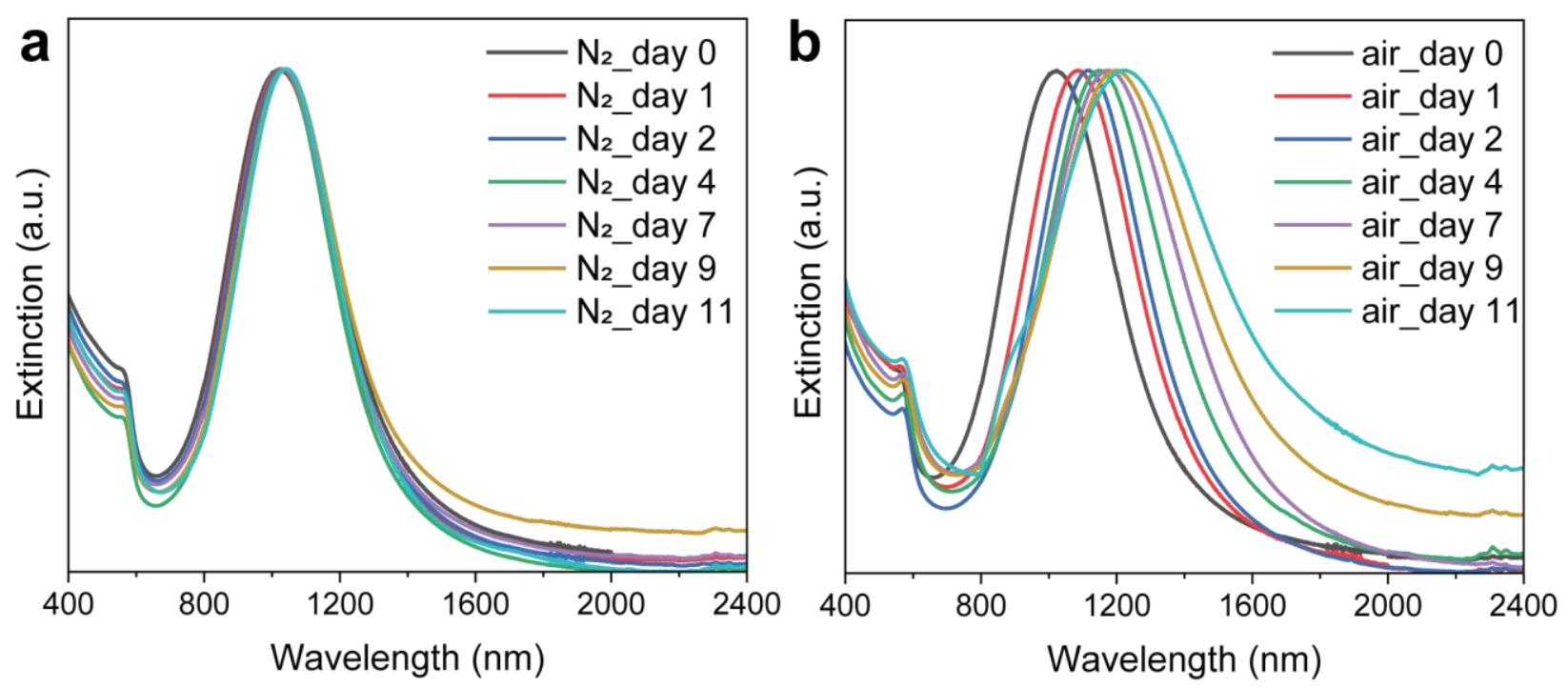

Figure S14. Time-dependent UV-Vis-NIR spectra of $\mathrm{Cu}$ nanorods dissolved in anhydrous toluene and stored (a) under a $\mathrm{N}_{2}$ atmosphere and (b) in air. 

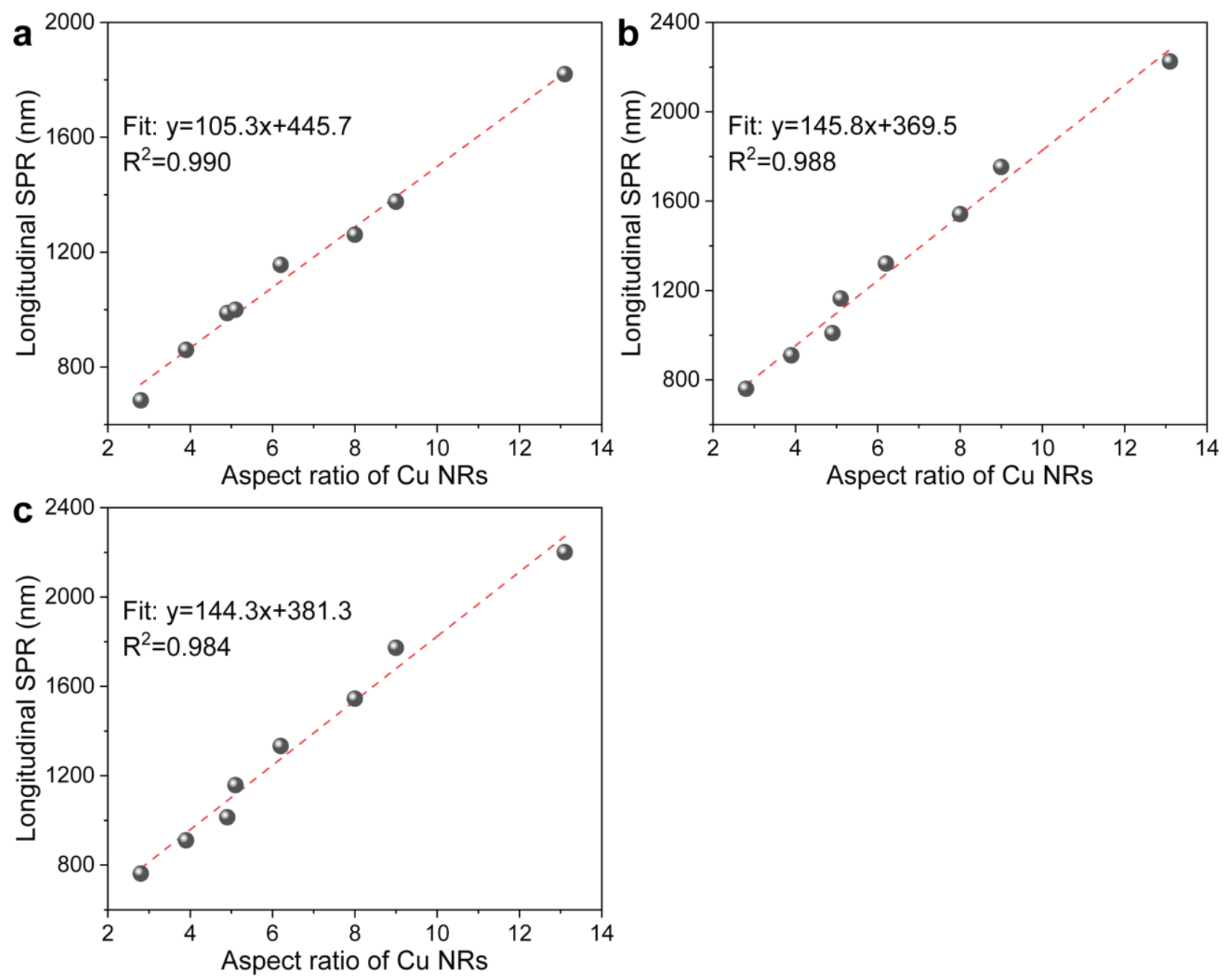

Figure S15. Dependence of longitudinal SPR peak wavelength on the aspect ratio of (a) pure $\mathrm{Cu}$ NRs (FDTD simulated), (b) $\mathrm{Cu} @ \mathrm{Cu}_{2} \mathrm{O}$ NRs (FDTD simulated), and (c) as-synthesized Cu NRs (experimental). The red dashed lines represent the linear fits to the data. 


\section{Mechanistic Studies of Cu NR Growth}
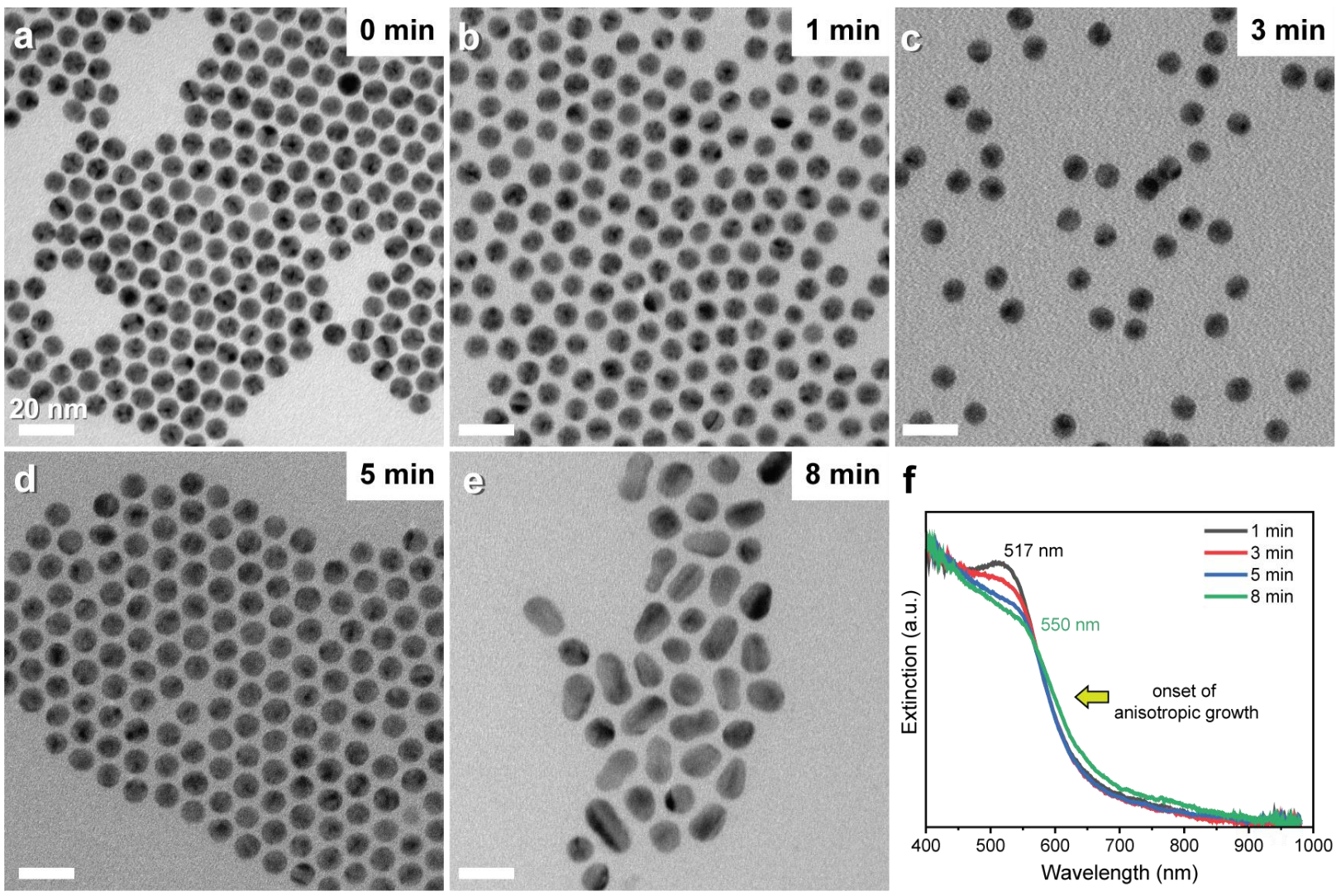

Figure S16. (a-e) Representative TEM images of reaction intermediates sampled during the early stages of $\mathrm{Cu}$ NR formation. (f) UV-Vis extinction spectra of different reaction intermediates. Scale bars: $20 \mathrm{~nm}$. 


\section{$3 \mathrm{~min}$}
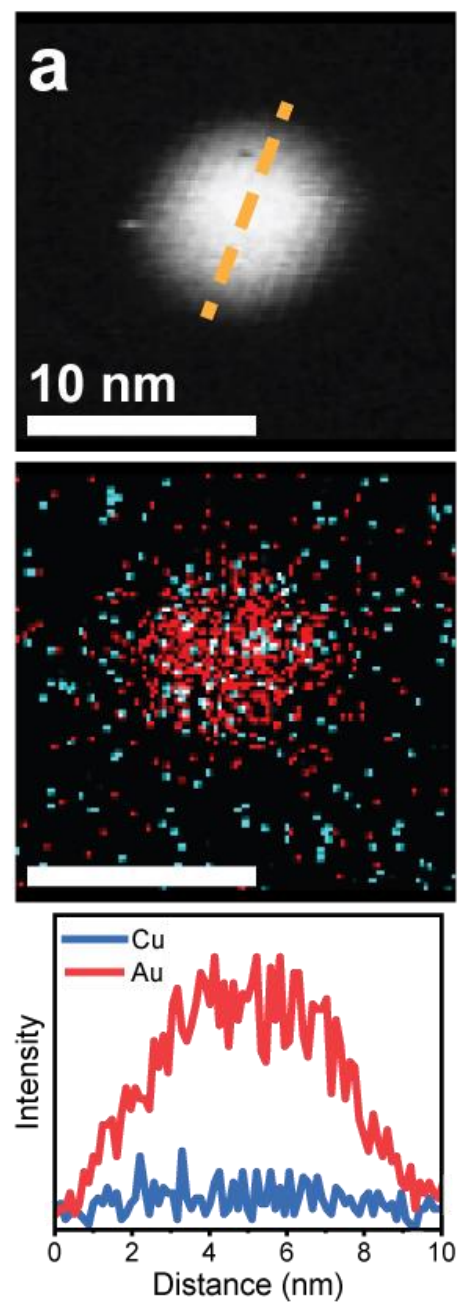

$5 \mathrm{~min}$
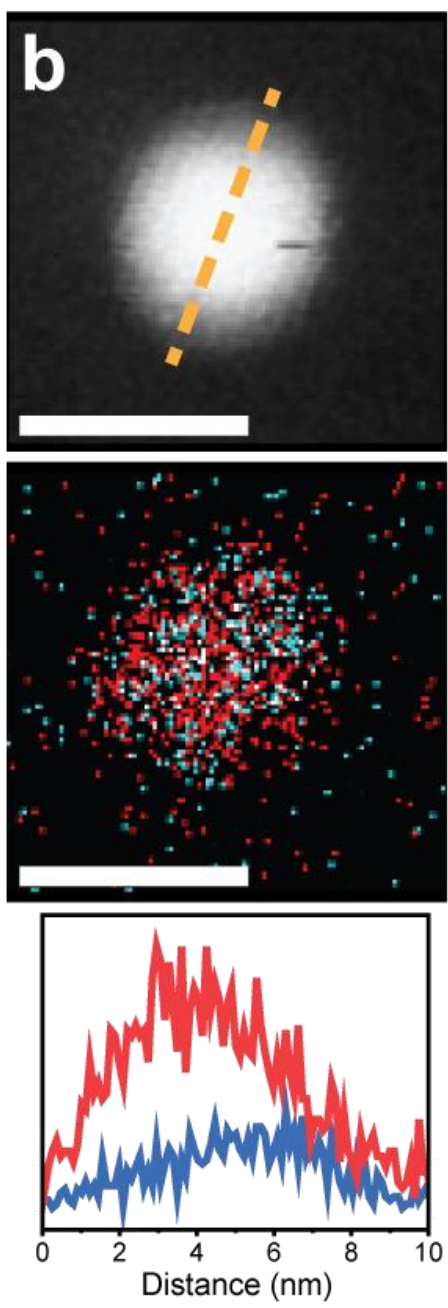

$8 \mathrm{~min}$
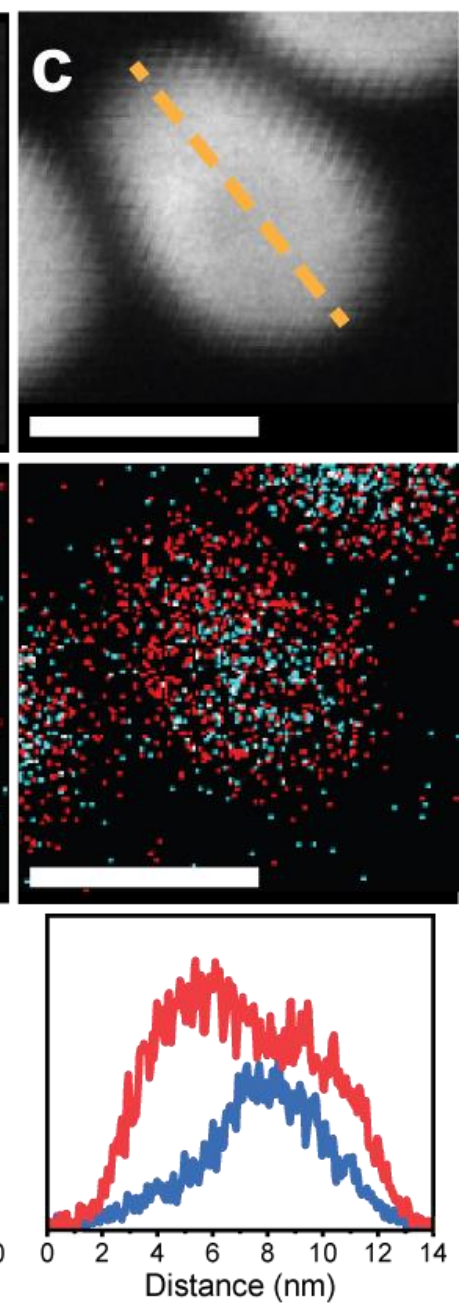

Figure S17. STEM-EDS analysis results of early-stage reaction intermediates sampled at (a) 3 min, (b) 5 min, and (c) 8 min. HAADF-STEM images (top row), STEM-EDS elemental maps (middle row), and STEM-EDS line profiles of $\mathrm{Cu}$ and $\mathrm{Au}$ contents along the yellow dashed lines (bottom row) are shown. 


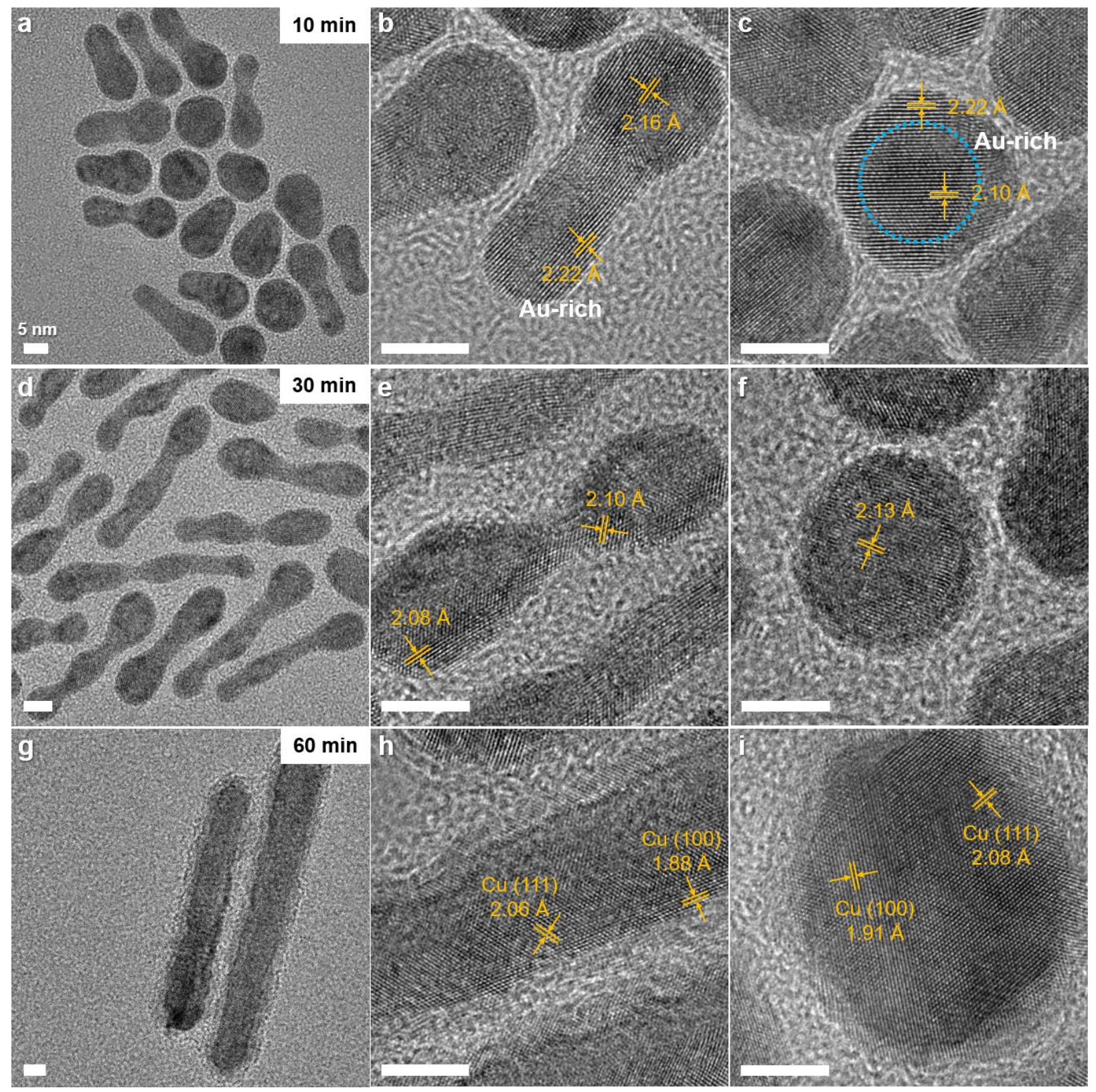

Figure S18. HRTEM images of reaction intermediates sampled at $10 \mathrm{~min}(\mathrm{a}-\mathrm{c}), 30 \mathrm{~min}(\mathrm{~d}-\mathrm{f})$ and 60 min (g-i) during the synthesis of Cu NRs. Both rod-shaped NCs (b,e,h) and spherical NCs (c,f,i) were analyzed for different reaction aliquots. At $10 \mathrm{~min}$, the $\{111\}$ interplanar spacings observed for both rod-shaped and spherical NCs were larger on NC surface than interior, suggesting a goldrich surface $\left(\mathrm{cf} . \mathrm{d}_{\mathrm{Cu}(111)}=2.09 \AA, \mathrm{d}_{\mathrm{Au}(111)}=2.36 \AA\right)$. After 30 min of reaction, the $\{111\}$ interplanar spacings were found to be essentially identical to bulk $\mathrm{Cu}\{111\}$. Scale bars: $5 \mathrm{~nm}$. 


\section{Determination of the Au content in Cu NRs based on XRD peak analysis and Vegard's law}

First, peaks in the XRD profiles were fitted by using the pesudo-Viogt function to retrieve the position of individual diffraction peaks. Next, the lattice constant a was calculated according to Bragg's law by using the two equations shown below. Finally, the average lattice constant was used to determine the atomic ratios of $\mathrm{Au}$ and $\mathrm{Cu}$ according to Vegard's law.

$$
d=\frac{2 d \sin \theta=\lambda}{\sqrt{h^{2}+k^{2}+l^{2}}}
$$

Table S3. Summary of lattice constants calculated from individual XRD peak positions for reaction intermediates sampled at different times.

\begin{tabular}{cccccccc}
\hline Time & \multicolumn{6}{c}{$2 \theta\left(^{\circ}\right)$ of XRD peak and derived lattice constant a $(\AA)$} \\
\cline { 2 - 8 }$(\mathrm{min})$ & $(111)$ & $\mathrm{a}(\AA)$ & $(200)$ & $\mathrm{a}(\AA)$ & $(220)$ & $\mathrm{a}(\AA)$ & Average a $(\AA)$ \\
\hline 10 & 41.575 & 3.759 & 48.287 & 3.767 & 71.071 & 3.749 & 3.758 \\
20 & 42.162 & 3.709 & 49.032 & 3.713 & 71.983 & 3.707 & 3.710 \\
30 & 42.805 & 3.656 & 49.606 & 3.672 & 73.132 & 3.657 & 3.662 \\
60 & 43.016 & 3.639 & 50.111 & 3.638 & 73.590 & 3.638 & 3.638 \\
\hline
\end{tabular}
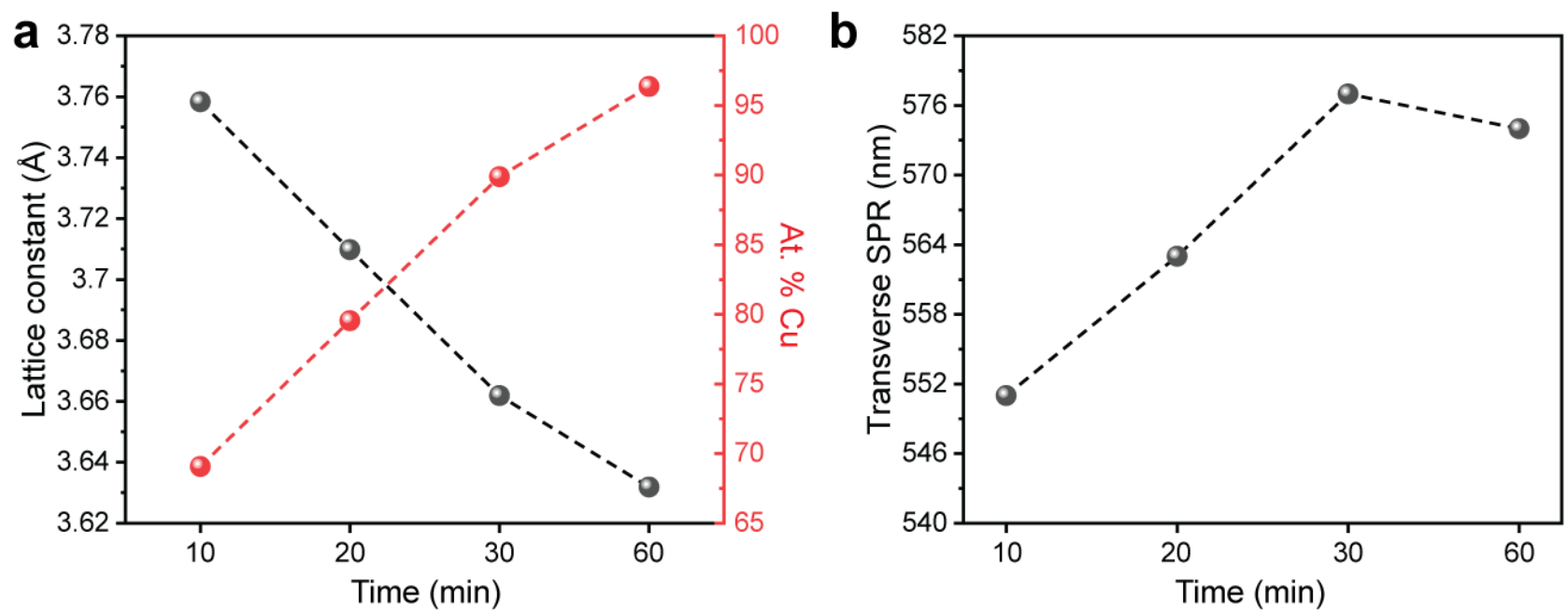

Figure S19. (a) Plot of time-dependent lattice constants (black dots) for reaction intermediates of $\mathrm{Cu}$ NRs synthesized using $9 \mathrm{~nm}$ Au seeds and their Au contents (red dots) determined according to Vegard's law. (b) Time-dependent evolution of transverse SPR peak position during synthesis of $\mathrm{Cu}$ NRs. 


\section{Estimation of the Au content in Cu NRs.}

We estimate the Au content in final $\mathrm{Cu}$ NRs under the assumptions that 1) each $\mathrm{Cu}$ NR derives from a single $\mathrm{Au}$ seed $\mathrm{NC}$, and 2) all $\mathrm{Au}$ atoms from seed $\mathrm{NCs}$ are retained in $\mathrm{Cu}$ NRs (i.e. no leaching of $\mathrm{Au}$ atoms). The dimensions of $\mathrm{Cu}$ NRs were measured from TEM images (Figure $\mathrm{S} 20 \mathrm{a})$, and the diameter of Au NC seeds was $9 \mathrm{~nm}$. Detailed results are summarized in Table S4. Below we provide an example of calculating the atomic ratio of gold in $\mathrm{Cu}$ NRs with the AR of 8.0. List of parameters used: $\mathrm{d}_{\mathrm{Cu}}=8.96 \mathrm{~g} / \mathrm{cm}^{3}, \mathrm{~d}_{\mathrm{Au}}=19.3 \mathrm{~g} / \mathrm{cm}^{3}$, molar mass of $\mathrm{Cu}=63.546 \mathrm{~g} / \mathrm{mol}$, molar mass of $\mathrm{Au}=196.967 \mathrm{~g} / \mathrm{mol}$.

Volume of Au NC seeds $V_{A u N C}=\frac{4}{3} \pi\left(\frac{9}{2}\right)^{3}=381.7 \mathrm{~nm}^{3}=3.817 \times 10^{-19} \mathrm{~cm}^{3}$

Number of atoms per $A u N C=\frac{\left(3.817 \times 10^{-19} \mathrm{~cm}^{3}\right) \times \frac{19.3 \mathrm{~g}}{\mathrm{~cm}^{3}}}{196.967 \frac{\mathrm{g}}{\mathrm{mol}}} \times 6.022 \times 10^{23} \mathrm{~mol}^{-1}$

$$
=22523
$$

Assuming a spherocylinder shape, the volume of a single $\mathrm{Cu}$ NR can be calculated as:

$V_{N R}=\frac{4}{3} \pi\left(\frac{12.5}{2}\right)^{3}+\pi\left(\frac{12.5}{2}\right)^{2} \times 88.5=1023+10738=11761 \mathrm{~nm}^{3}$

The number of $\mathrm{Cu}$ atoms within each NR can be obtained by subtracting the volume of $\mathrm{Au}$ from that of $\mathrm{Cu} \mathrm{NR}$ :

$$
\begin{aligned}
\text { Number of Cu atoms } & =\frac{\left(11761-381.7 \mathrm{~nm}^{3}\right) \times 8.96 \frac{\mathrm{g}}{\mathrm{cm}^{3}}}{63.546 \frac{\mathrm{g}}{\mathrm{mol}}} \times 6.022 \times 10^{23} \mathrm{~mol}^{-1} \\
& =966179
\end{aligned}
$$

Consequently, At. $\%$ Cu $=\frac{966179}{22523+966179} \times 100=97.7$

$$
\text { At. } \% A u=\frac{22523}{22523+966179} \times 100=2.3
$$


a

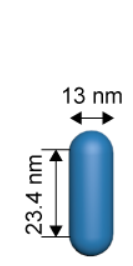

AR: 2.8

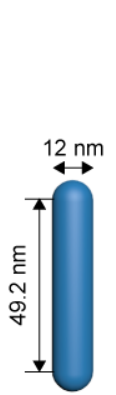

5.1

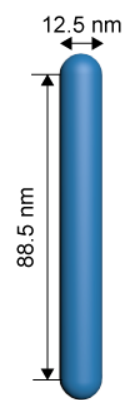

8.0

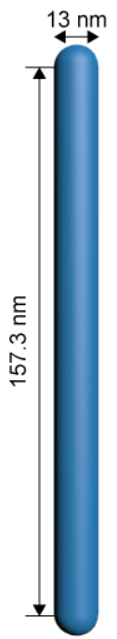

13.1

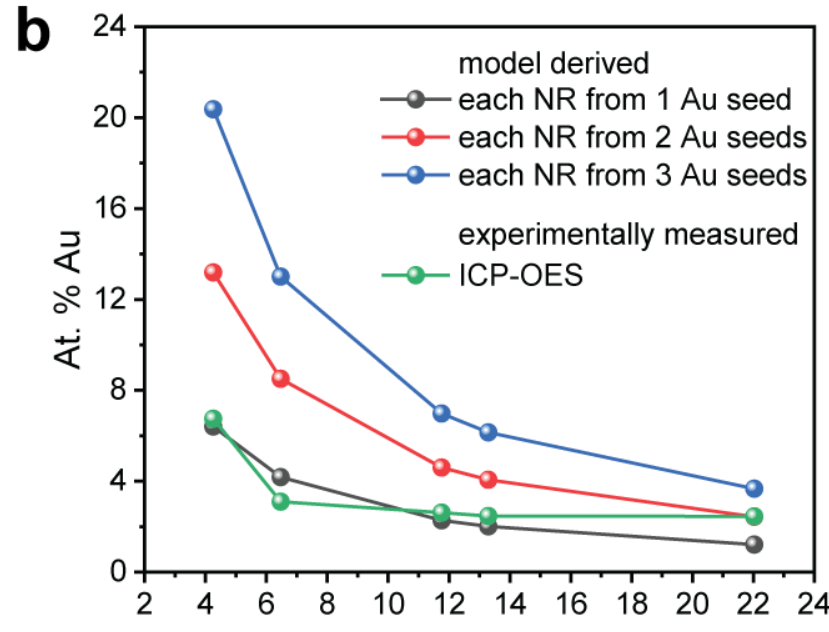

Volume $\left(\mathrm{nm}^{3}\right)$

Figure S20. (a) Dimensions of different $\mathrm{Cu}$ NRs obtained from TEM image analysis. (b) Calculated $\mathrm{Au}$ content versus the total volume of $\mathrm{Cu}$ NRs shown in (a), assuming that each $\mathrm{Cu} \mathrm{NR}$ derives from a single (black dots), two (red dots), and three Au NC seeds (blue dots) and all Au atoms from the seeds are retained in the final $\mathrm{Cu}$ NRs. The actual $\mathrm{Au}$ contents of different $\mathrm{Cu}$ NRs synthesized after one hour of reaction were quantified by using ICP-OES (green dots).

Table S4. Detailed calculations of the $\mathrm{Cu}$ and $\mathrm{Au}$ atomic ratios for different $\mathrm{Cu} \mathrm{NRs}$.

\begin{tabular}{|c|c|c|c|c|c|}
\hline & \multicolumn{5}{|c|}{ Aspect ratio } \\
\hline & 2.8 & 5.1 & 8.0 & 9.0 & 13.1 \\
\hline$\left.V_{A u ~ N C}\left(n^{3}\right)^{3}\right)$ & & & 382 & & \\
\hline Number of Au atoms & & & $2.3 \times 10^{4}$ & & \\
\hline Diameter (nm) & 13.0 & 12.0 & 12.5 & 12.5 & 13.0 \\
\hline Length (nm) & 36.4 & 61.2 & 100 & 112.5 & 170.3 \\
\hline Lcylinder (nm) & 23.4 & 49.2 & 88.5 & 90 & 157.3 \\
\hline$V_{\text {spherical caps }}\left(\mathrm{nm}^{3}\right)$ & 1150 & 905 & 1023 & 1023 & 1150 \\
\hline$V_{\text {cylinder }}\left(\mathbf{n m}^{3}\right)$ & 3106 & 5564 & 10738 & 12272 & 20879 \\
\hline$V_{C u}\left(n^{3}\right)$ & 3875 & 6087 & 11379 & 12913 & 21647 \\
\hline Number of $\mathrm{Cu}$ atoms & $3.3 \times 10^{5}$ & $5.2 \times 10^{5}$ & $9.7 \times 10^{5}$ & $1.1 \times 10^{6}$ & $1.8 \times 10^{6}$ \\
\hline At. $\% \mathrm{Cu}$ & 93.6 & 95.8 & 97.7 & 98.0 & 98.8 \\
\hline At. \% Au & 6.4 & 4.2 & 2.3 & 2.0 & 1.2 \\
\hline
\end{tabular}


Table S5. ICP-OES analysis results of the $\mathrm{Au}$ and $\mathrm{Cu}$ contents of the reaction solution extracted at different times.

\begin{tabular}{ccccc}
\hline $\begin{array}{c}\text { Reaction time } \\
\text { (min) }\end{array}$ & Metal content of supernatant & $\begin{array}{c}\text { Mass of Cu in so- } \\
\text { lution normalized } \\
\text { by Cu in reaction } \\
\text { precursor }\end{array}$ & $\begin{array}{c}\text { Mass of Au in so- } \\
\text { lution normal- } \\
\text { ized by Au in } \\
\text { seed NCs }\end{array}$ \\
\hline $\mathbf{1 5}$ & 99.995 & At. \% Au & $31.69 \%$ & $0.049 \%$ \\
$\mathbf{3 0}$ & 99.981 & 0.019 & $12.93 \%$ & $0.065 \%$ \\
$\mathbf{6 0}$ & 99.980 & 0.020 & $1.83 \%$ & $0.009 \%$ \\
\hline
\end{tabular}

Notes: (1) ICP-OES analysis indicated that dissolution or leaching of Au atoms from seed NCs into the reaction solution is insignificant.

(2) ICP-OES analysis also revealed that more than $98 \%$ of $\mathrm{Cu}$ atoms in the precursor (i.e., $\mathrm{CuCl}_{2} \cdot 2 \mathrm{H}_{2} \mathrm{O}$ ) end up in final nanorods, suggesting that this synthesis method has high atom economy. 


\section{Major products}
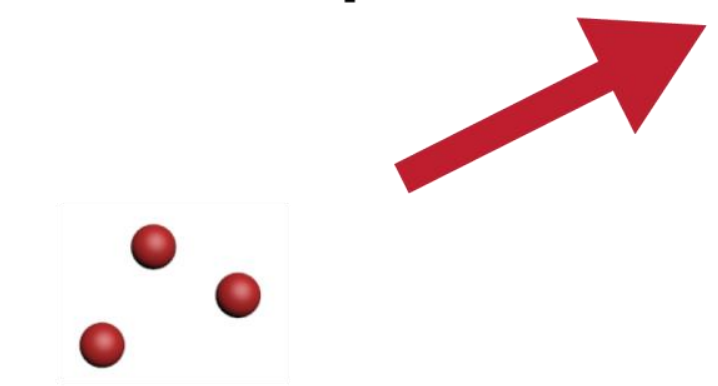

Au NC seeds

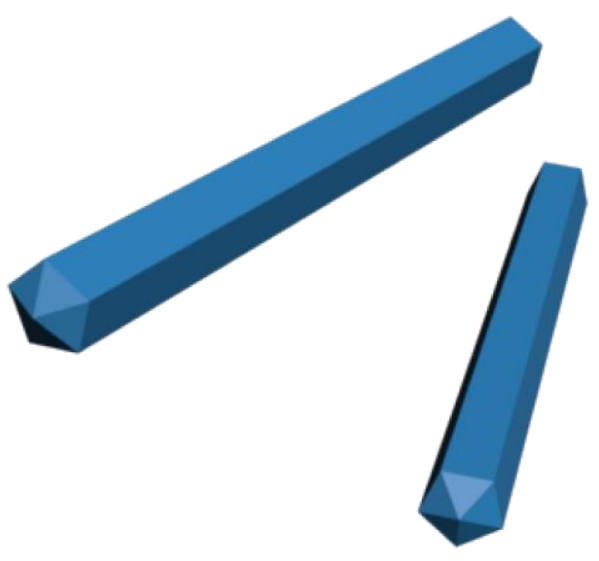

Nanorods
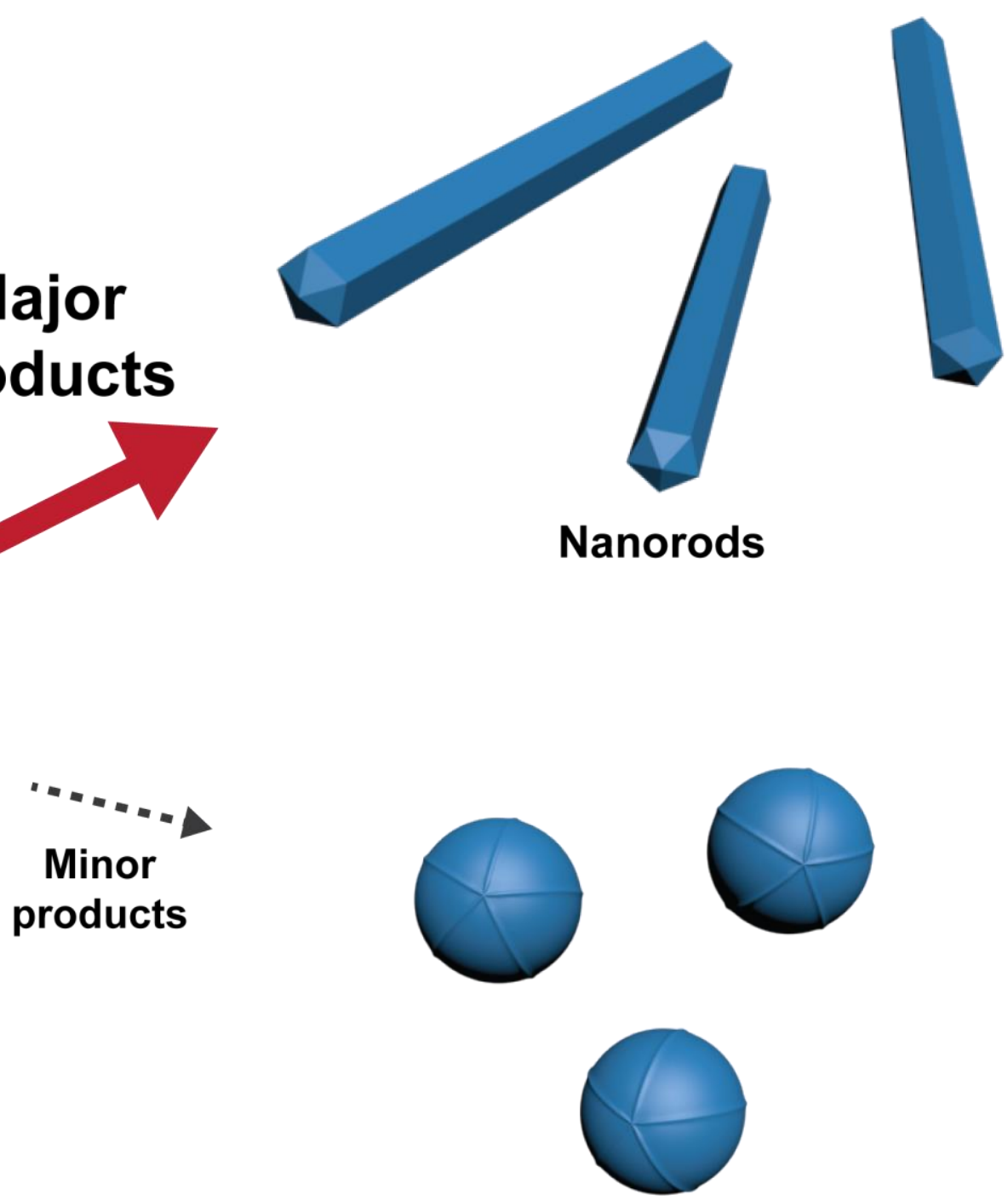

Nanospheres (side products)

Figure S21. Schematic illustration of rod-shaped and spherical NC products obtained from Cu NR synthesis. 

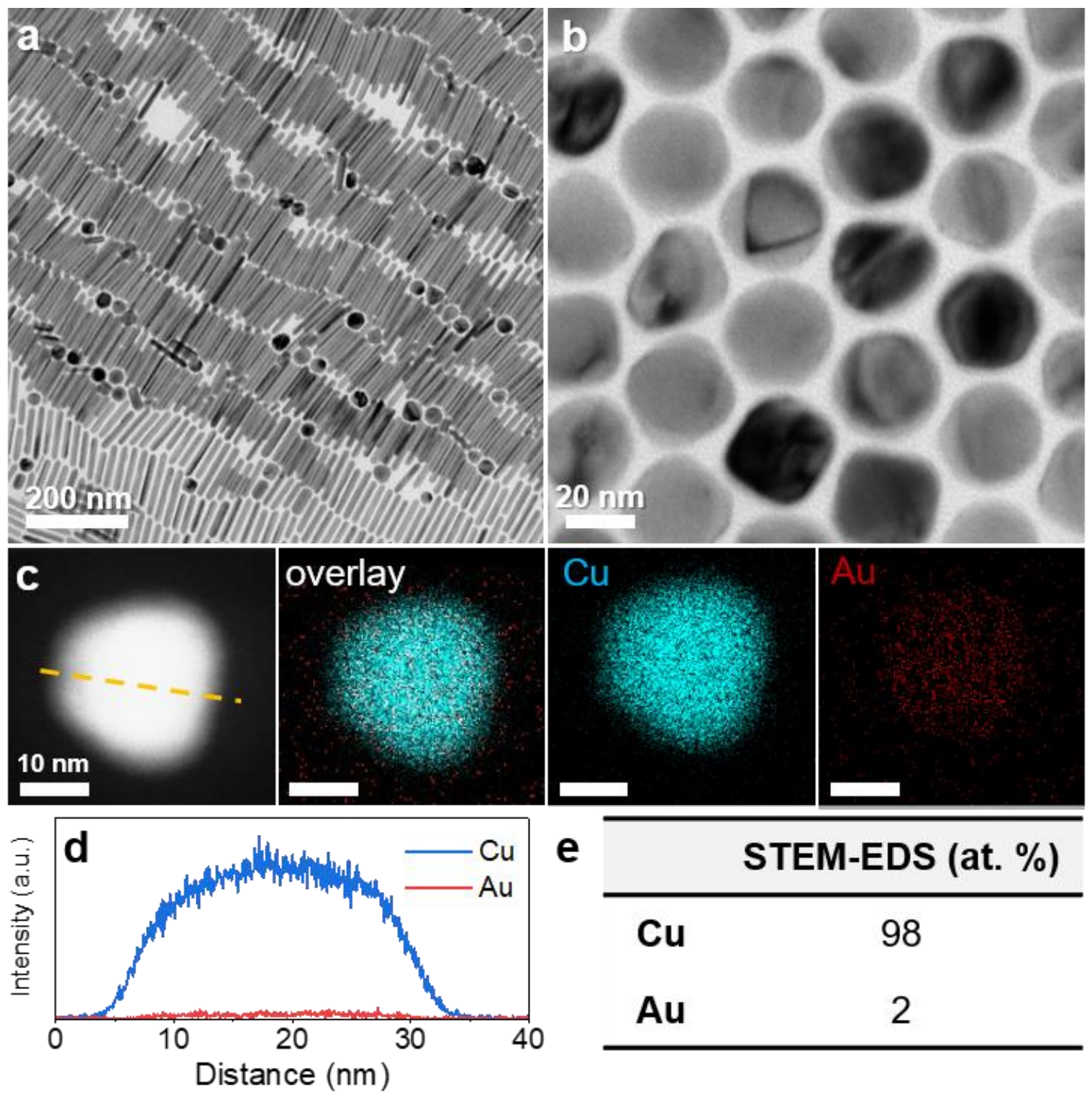

Figure S22. (a) Representative TEM image of (a) crude $\mathrm{Cu}$ NR products (average AR: 8.0) and (b) spherical NC impurities isolated via size-selective purification. (c) HAADF-STEM image and STEM-EDS elemental maps of a spherical NC produced during $\mathrm{Cu}$ NR growth. (d) STEM-EDS line profile showing the variation of $\mathrm{Cu}$ and $\mathrm{Au}$ contents along the yellow dashed line indicated in (c). (e) Summary of $\mathrm{Cu}$ :Au atomic ratios determined from STEM-EDS mapping. 


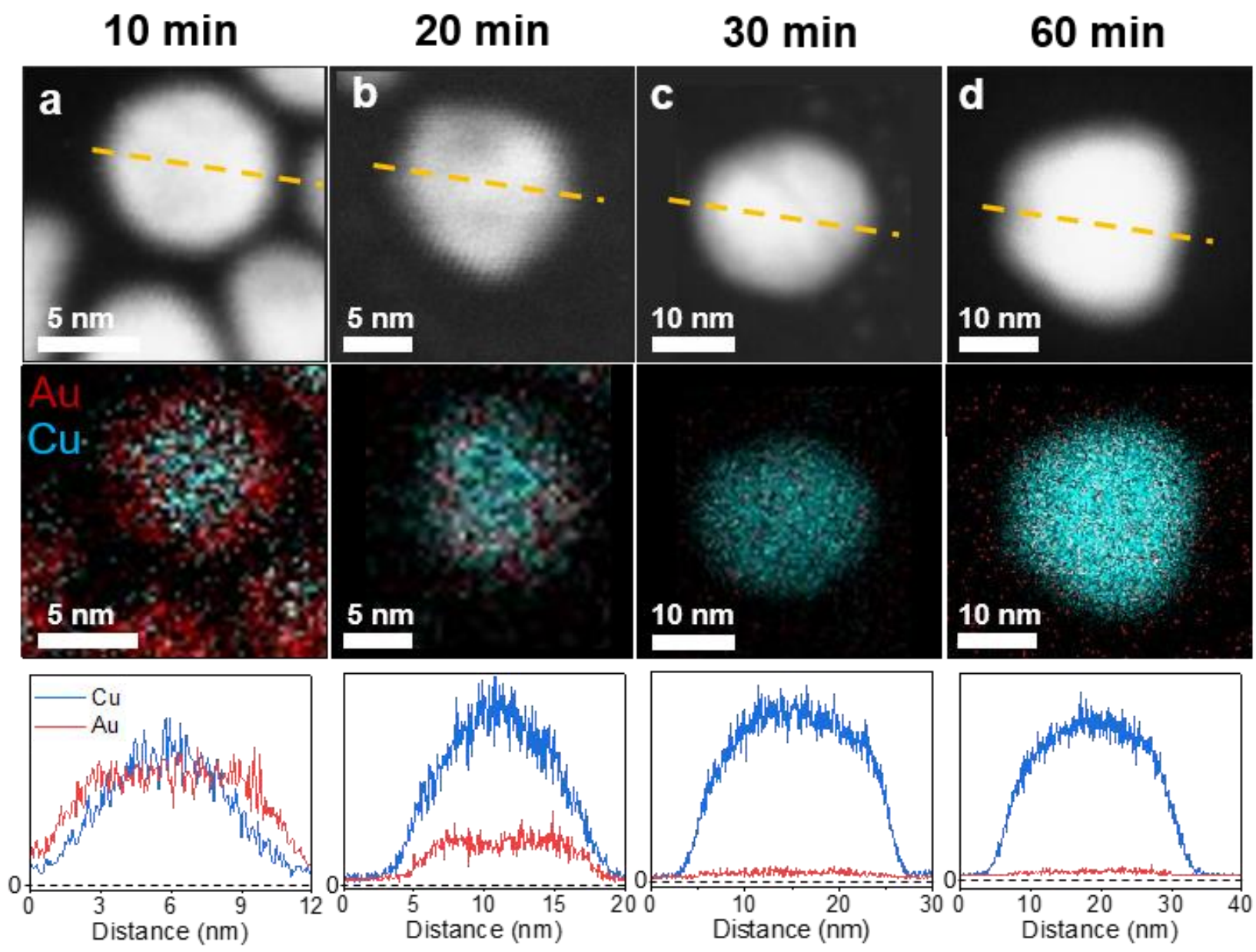

Figure S23. STEM-EDS elemental mapping results of spherical NCs produced at (a) $10 \mathrm{~min}$, (b) $20 \mathrm{~min}$, (c) $30 \mathrm{~min}$, and (d) $60 \mathrm{~min}$ during Cu NR growth. At $10 \mathrm{~min}$, AuCu alloy NCs with a goldrich shell predominated. These spherical NCs continued to grow via incorporation of $\mathrm{Cu}$ atoms and after $30 \mathrm{~min}$ of reaction, the residual Au content was determined to be less than 5\%. STEMEDS line profiles in the bottom row show the variation of $\mathrm{Cu}$ and $\mathrm{Au}$ contents along the yellow dashed line indicated on the corresponding HAADF-STEM image. 


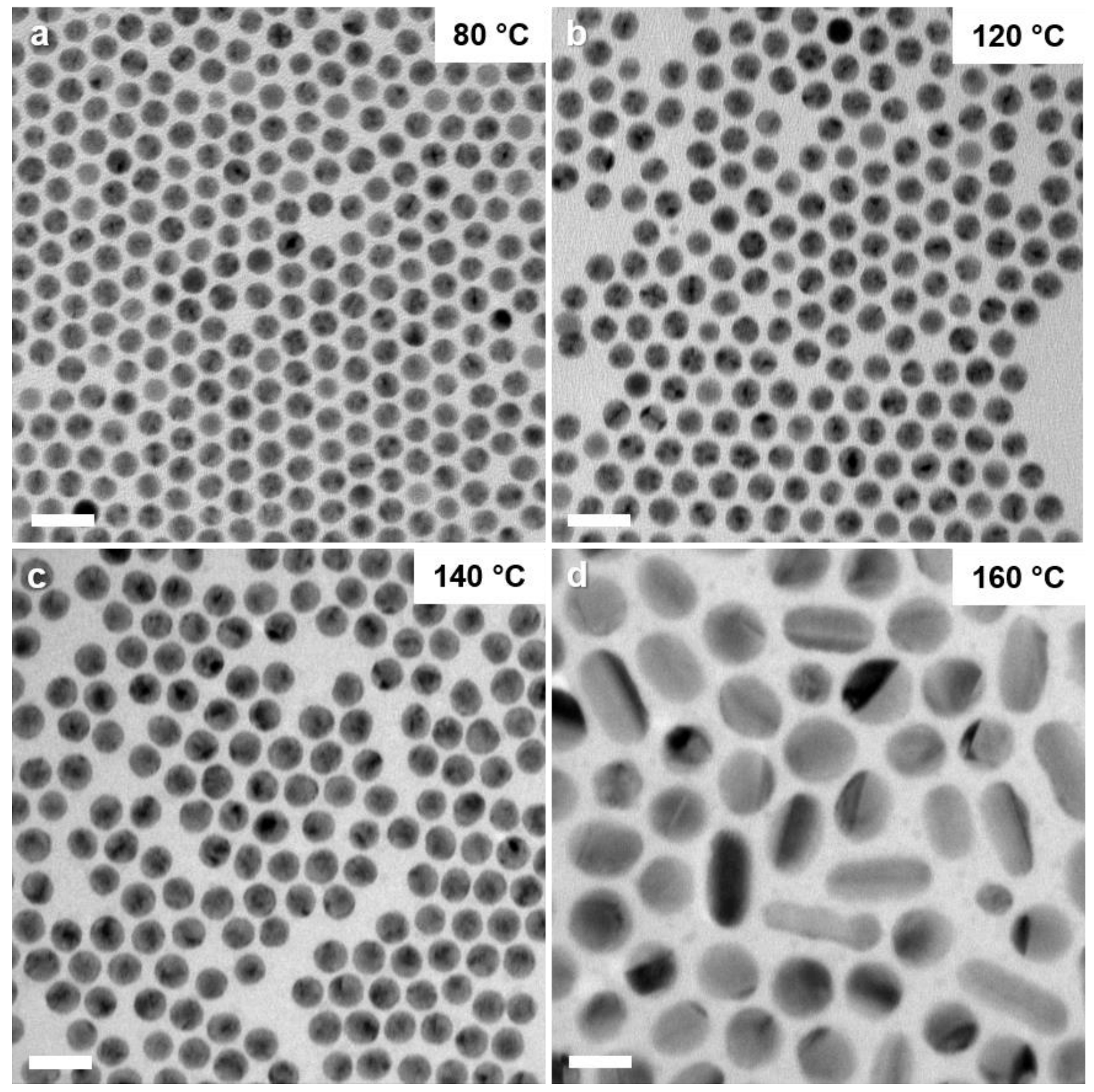

Figure S24. TEM images of NCs obtained after performing the same synthesis as Cu NRs except for lower reaction temperatures: (a) $80{ }^{\circ} \mathrm{C}$, (b) $120{ }^{\circ} \mathrm{C}$, (c) $140{ }^{\circ} \mathrm{C}$, and (d) $160{ }^{\circ} \mathrm{C}$. The diameter of spherical NCs are: (a) $9.3 \pm 0.4 \mathrm{~nm}$, (b) $10.0 \pm 0.5 \mathrm{~nm}$, (c) $11.2 \pm 0.5 \mathrm{~nm}$, and (d) $18 \pm 1.6 \mathrm{~nm}$. The level of $\mathrm{Cu}$ incorporation into $\mathrm{Au}$ seed NCs was found to increase for increasing temperatures, yet anisotropic growth was not observed below $160{ }^{\circ} \mathrm{C}$. Scale bars: $20 \mathrm{~nm}$. 

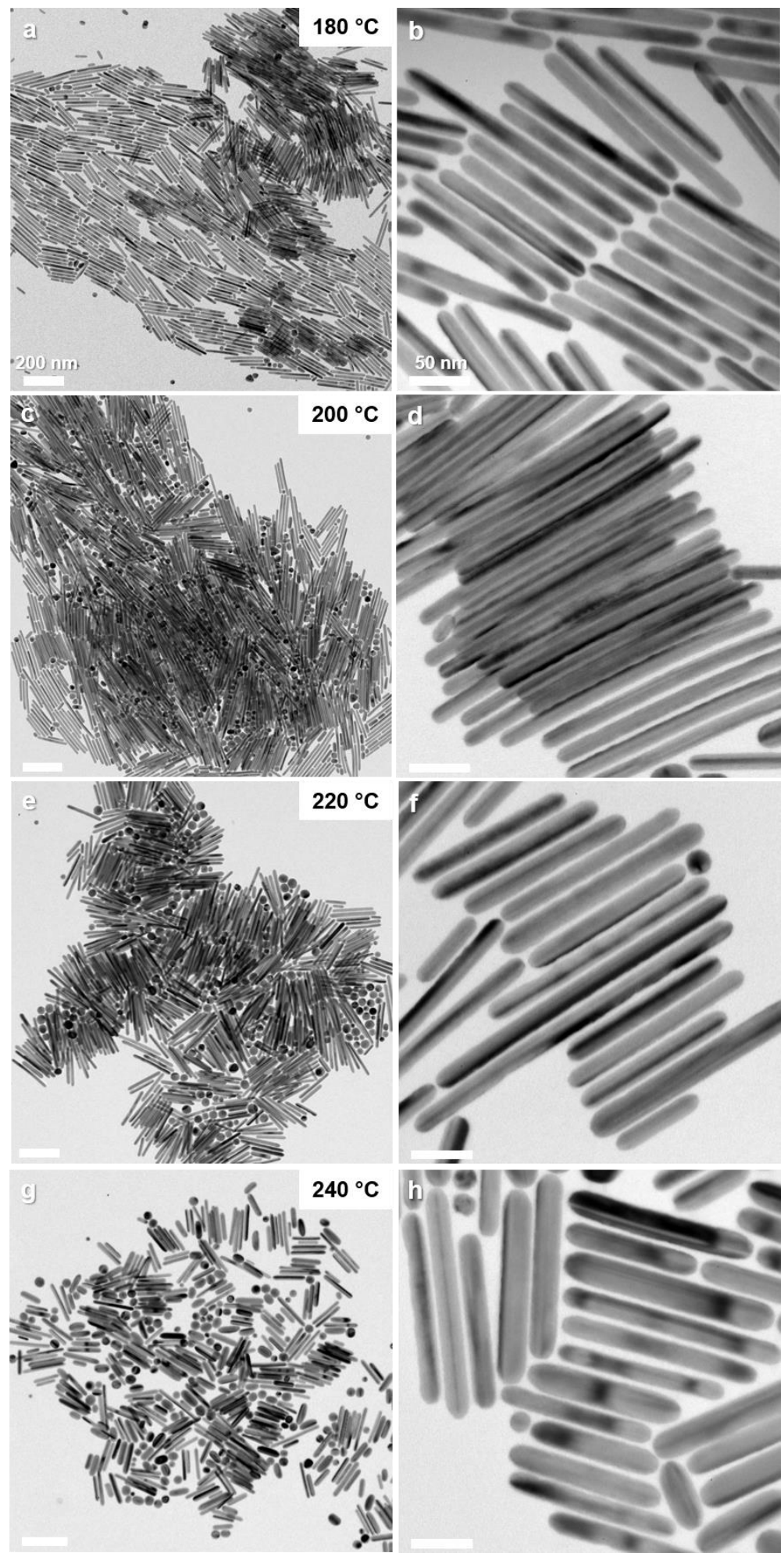

Figure S25. TEM images of $\mathrm{Cu}$ NRs obtained when the reaction was carried out at (a,b) $180{ }^{\circ} \mathrm{C}$, (c,d) $200{ }^{\circ} \mathrm{C}$, (e,f) $220{ }^{\circ} \mathrm{C}$, and $(\mathrm{g}, \mathrm{h}) 240{ }^{\circ} \mathrm{C}$. When the reaction temperature exceeded $200{ }^{\circ} \mathrm{C}$, polydisperse $\mathrm{Cu}$ NRs together with a modest amount of spherical NCs were obtained. Scale bars: $200 \mathrm{~nm}$ (images in the left column), $50 \mathrm{~nm}$ (images in the right column). 


\section{Effects of Size of Au Seeds on Cu NR Growth}

The diameter of $\mathrm{Au}$ seeds was varied to assess its impacts on formation kinetics and dimensions of Cu NRs (Figure S26-S27, Table S6-S7). As shown in Table S8 and Figure S28, OLAM-capped $\mathrm{Au}$ NCs in the diameter range of 5-9 nm offered a comparable level of size uniformity and AR tunability, with less seeds consistently producing NRs of higher ARs. STEM-EDS characterization showed that the kinetic pathway of NR formation was essentially identical among different seed sizes (Figure S29-S31). Moreover, early-stage (e.g., $10 \mathrm{~min}$ ) $\mathrm{Cu}$ incorporation appeared to be more facile with larger $\mathrm{Au}$ seeds, yet the atomic ratio of $\mathrm{Cu}$ all converged to ca. 98 at. \% after 60 min of reaction (Figure S32).

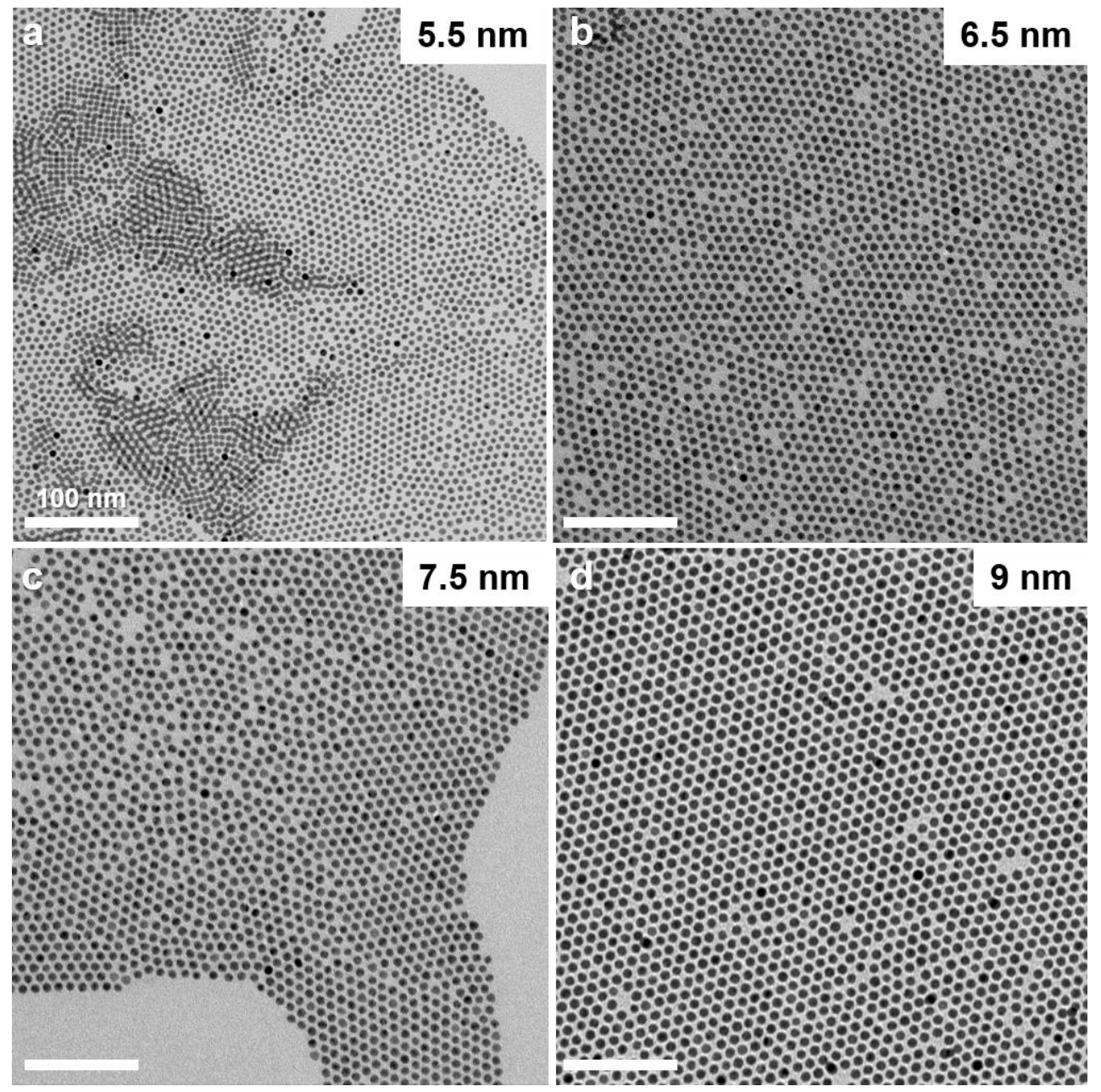

Figure S26. TEM images of Au seed NCs with an average diameter of (a) $5.5 \mathrm{~nm}$, (b) $6.5 \mathrm{~nm}$, (c) $7.5 \mathrm{~nm}$, and (d) $9.0 \mathrm{~nm}$. The size of Au NCs was controlled by changing the amount of reducing agent (i.e., TBAB) and the reaction temperature as detailed in Table S6. Scale bars: $100 \mathrm{~nm}$. 
Table S6. Reaction conditions for the synthesis of different Au seeds.

\begin{tabular}{ccccccc}
\hline $\begin{array}{c}\text { Diameter } \\
(\mathrm{nm})\end{array}$ & $\begin{array}{c}\text { OLAM-TCI } \\
(\mathrm{mL})\end{array}$ & $\begin{array}{c}\text { Toluene } \\
(\mathrm{mL})\end{array}$ & $\begin{array}{c}\mathbf{H A u C l}_{4} \\
(\mathrm{mmol})\end{array}$ & $\begin{array}{c}\text { TBAB } \\
(\mathrm{mmol})\end{array}$ & $\begin{array}{c}\text { Reaction } \\
\text { Temp. }\left({ }^{\circ} \mathrm{C}\right)\end{array}$ & $\begin{array}{c}\text { Time } \\
(\mathrm{hr})\end{array}$ \\
\hline 5.5 & 10 & 10 & 1 & 0.20 & 25 & 1 \\
6.5 & 10 & 10 & 1 & 0.25 & 20 & 1 \\
7.5 & 10 & 10 & 1 & 0.20 & 20 & 1 \\
9.0 & 10 & 10 & 1 & 0.25 & 15 & 1 \\
\hline
\end{tabular}

\section{Determination of Au NC concentrations from UV-Vis extinction spectra}

The amount of Au seeds was found to play a decisive role in controlling the aspect ratio of $\mathrm{Cu}$ NRs. Therefore, we used optical density (OD) to precisely quantify the concentration of Au NCs in solution. The size-dependent extinction coefficients $(\varepsilon)$ of spherical Au NCs were determined by performing linear regression to the data points reported previously (Figure S27, Table S7). ${ }^{13}$ To maintain the same number of seeds when working with differently sized Au NCs, we used Beer's law $\left(\mathrm{A}=\varepsilon^{*} \mathrm{~b}^{*} \mathrm{C}\right)$ and the size-dependent extinction coefficients to calculate the volume of $\mathrm{Au} \mathrm{NC}$ solution needed for synthesis (Table S8).

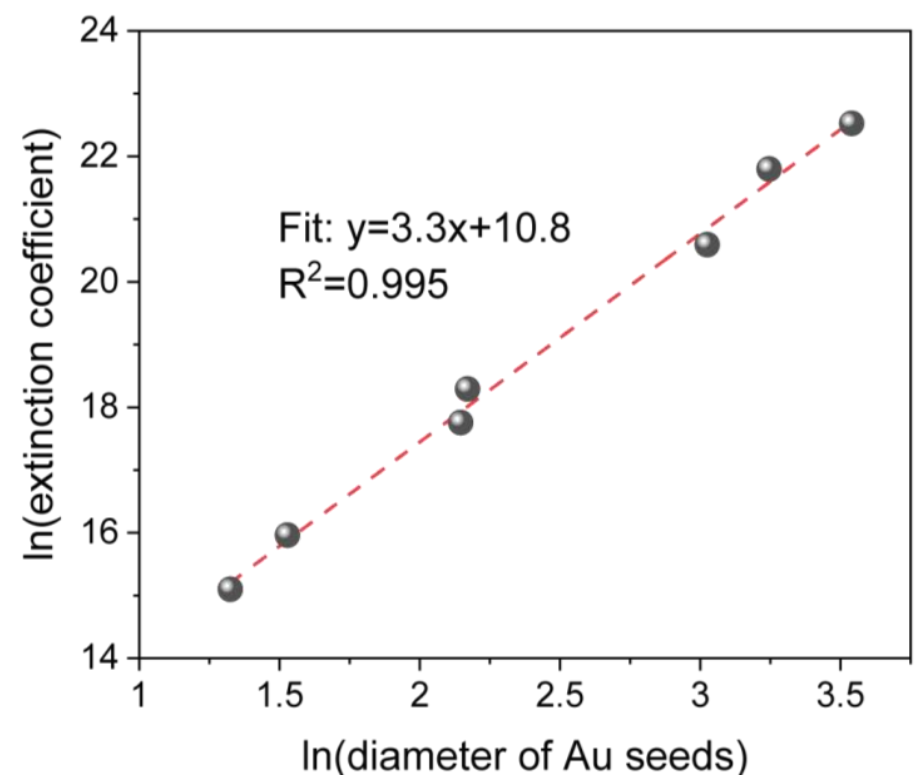

Figure S27. Plot (natural logarithmic scale) of size-dependent extinction coefficients of spherical Au NCs with diameters ranging from 3.76 to $34.46 \mathrm{~nm}$ as reported previously. ${ }^{13}$ The red dashed line represents the linear fit to the data. 
Table S7. Calculated extinction coefficients of Au NCs with diameters between 5.5 and $9.0 \mathrm{~nm}$ based on linear regression analysis shown in Figure S27.

\begin{tabular}{ccc}
\hline $\begin{array}{c}\text { NC diameter } \\
(\mathbf{n m})\end{array}$ & $\begin{array}{c}\text { Calculated } \\
\text { extinction coefficient } \\
\left(\mathbf{M}^{-1} \cdot \mathbf{c m}^{-1}\right)\end{array}$ & $\begin{array}{c}\text { Relative NC concentration } \\
\text { given the same OD }\end{array}$ \\
\hline 5.5 & $1.417 \times 10^{7}$ & 5.13 \\
6.5 & $2.467 \times 10^{7}$ & 2.95 \\
7.5 & $3.969 \times 10^{7}$ & 1.83 \\
9.0 & $7.273 \times 10^{7}$ & 1 \\
\hline
\end{tabular}

Table S8. Synthetic parameters of $\mathrm{Cu}$ NRs prepared by using differently sized Au seeds. Within each of the three categories, namely short, medium, and long $\mathrm{Cu} \mathrm{NRs}$, the injection volume of $\mathrm{Au}$ seed solution was varied to ensure that the same number of Au seeds was used.

\begin{tabular}{ccccc}
\hline & $\begin{array}{c}\text { Diameter of Au } \\
\text { seeds }(\mathbf{n m})\end{array}$ & $\begin{array}{c}\text { OD of Au } \\
\text { seed solution }\end{array}$ & $\begin{array}{c}\text { Injection volume } \\
\text { of Au seeds }(\mathbf{m L})\end{array}$ & $\begin{array}{c}\text { Aspect ratio } \\
\text { of Cu NR }\end{array}$ \\
\hline Short & 5.5 & 0.11 & 0.31 & $3.7 \pm 0.7$ \\
Cu NRs & 6.5 & 0.17 & 0.34 & $3.6 \pm 0.4$ \\
& 7.5 & 0.18 & 0.52 & $3.9 \pm 0.5$ \\
& 9.0 & 0.23 & 0.75 & $2.8 \pm 0.6$ \\
Medium & 5.5 & 0.11 & 0.20 & $5.3 \pm 1.3$ \\
Cu NRs & 6.5 & 0.17 & 0.23 & $4.9 \pm 0.8$ \\
& 7.5 & 0.18 & 0.35 & $6.2 \pm 1.6$ \\
& 9.0 & 0.23 & 0.5 & $5.1 \pm 1.4$ \\
\hline Long & 5.5 & 0.11 & 0.10 & $9.4 \pm 1.9$ \\
& 6.5 & 0.17 & 0.10 & $10.5 \pm 1.8$ \\
& 7.5 & 0.18 & 0.12 & $11.9 \pm 3.3$ \\
& 9.0 & 0.23 & 0.17 & $13.1 \pm 4.5$ \\
\hline
\end{tabular}




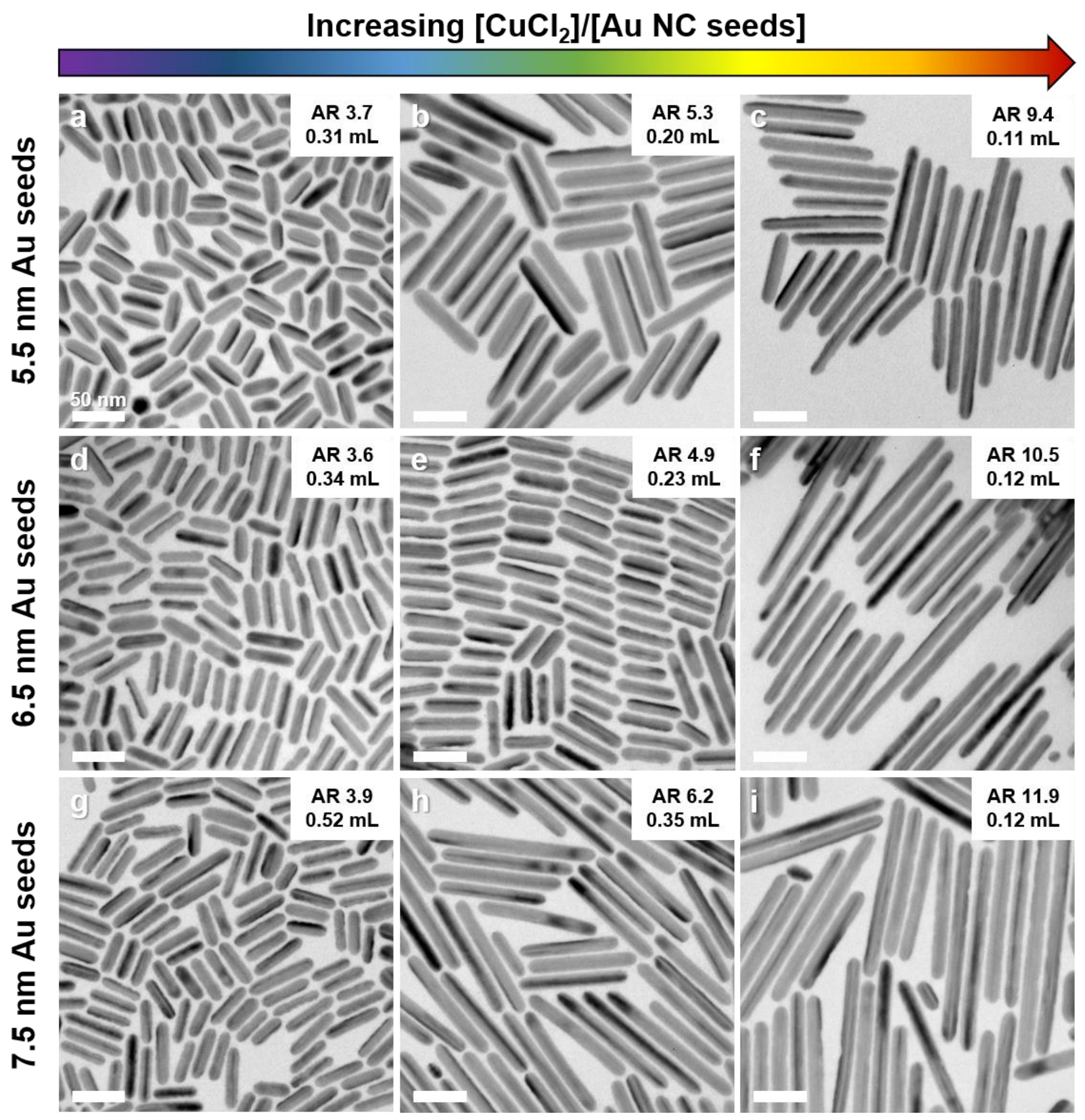

Figure S28. TEM images of Cu NRs synthesized by using Au seeds of different sizes. For reactions shown within each column, the number of Au NCs was kept identical by using different injection volumes as indicated in the legend. A monotonic increase in the aspect ratio of $\mathrm{Cu}$ NRs was observed upon increasing the $\left[\mathrm{CuCl}_{2}\right]$ :[Au seeds] ratio. Scale bars: $50 \mathrm{~nm}$. 


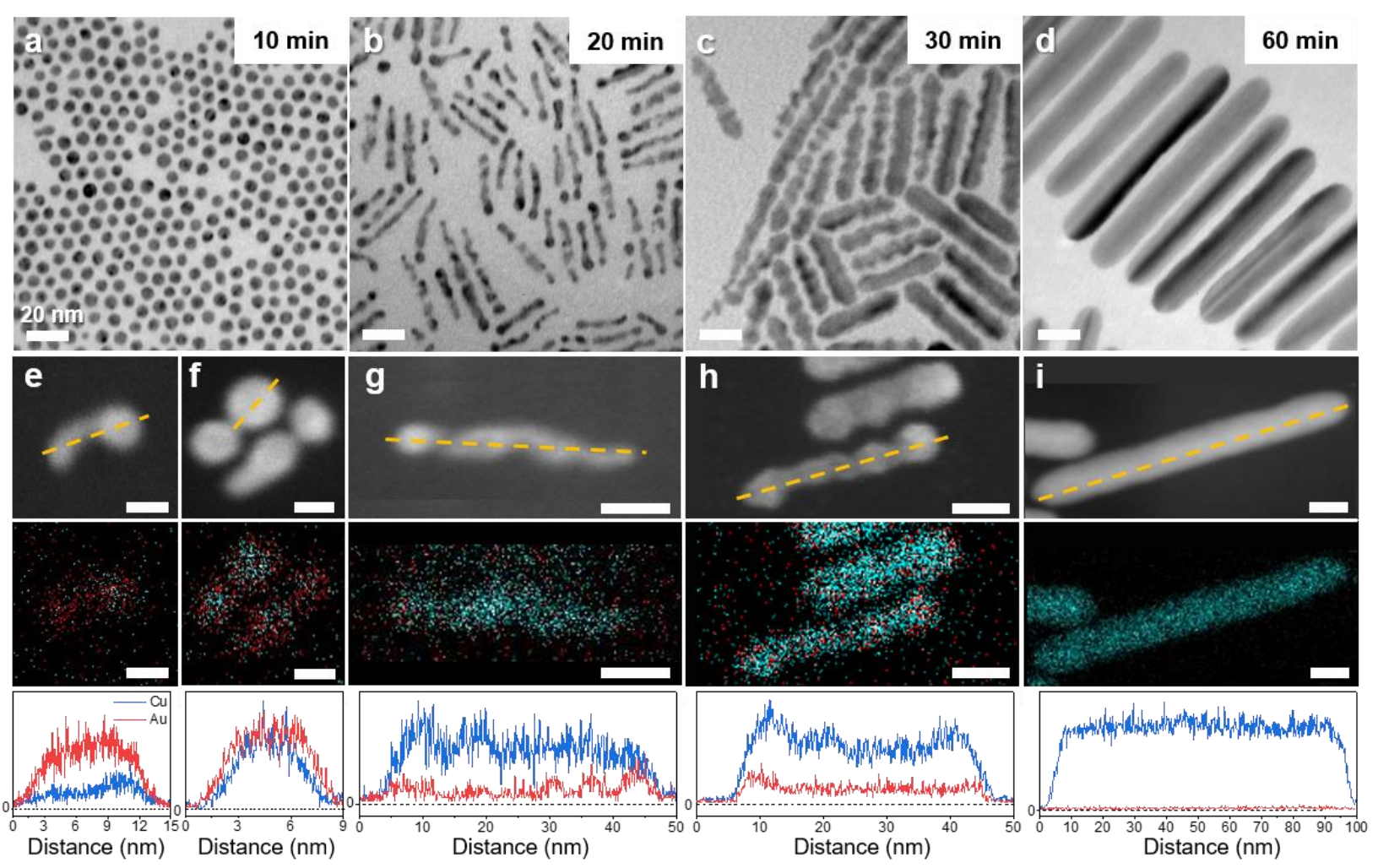

Figure S29. (a-d) TEM images and STEM-EDS analysis results of reaction intermediates of $\mathrm{Cu}$ NR synthesized by using $5.5 \mathrm{~nm}$ Au NCs as seeds. At $10 \mathrm{~min}$, a mixture of elongated (e) and spherical (f) NCs with a gold-rich surface was observed. At 20 min, zigzag-shaped NRs appeared $(\mathrm{b}, \mathrm{g})$. Between 20 and $30 \mathrm{~min}$, these NRs continued to grow via incorporation of more $\mathrm{Cu}$ atoms $(\mathrm{c}, \mathrm{h})$. Afterward, the surface of NRs became smoother as more $\mathrm{Cu}$ atoms were deposited, yielding highly uniform $\mathrm{Cu}$ NRs at $60 \mathrm{~min}(\mathrm{~d}$,i). STEM-EDS analysis revealed continuous enrichment of $\mathrm{Cu}$ concomitant with NR growth. Scale bars: $20 \mathrm{~nm}(\mathrm{a}-\mathrm{d}), 10 \mathrm{~nm}$ (e-i). 


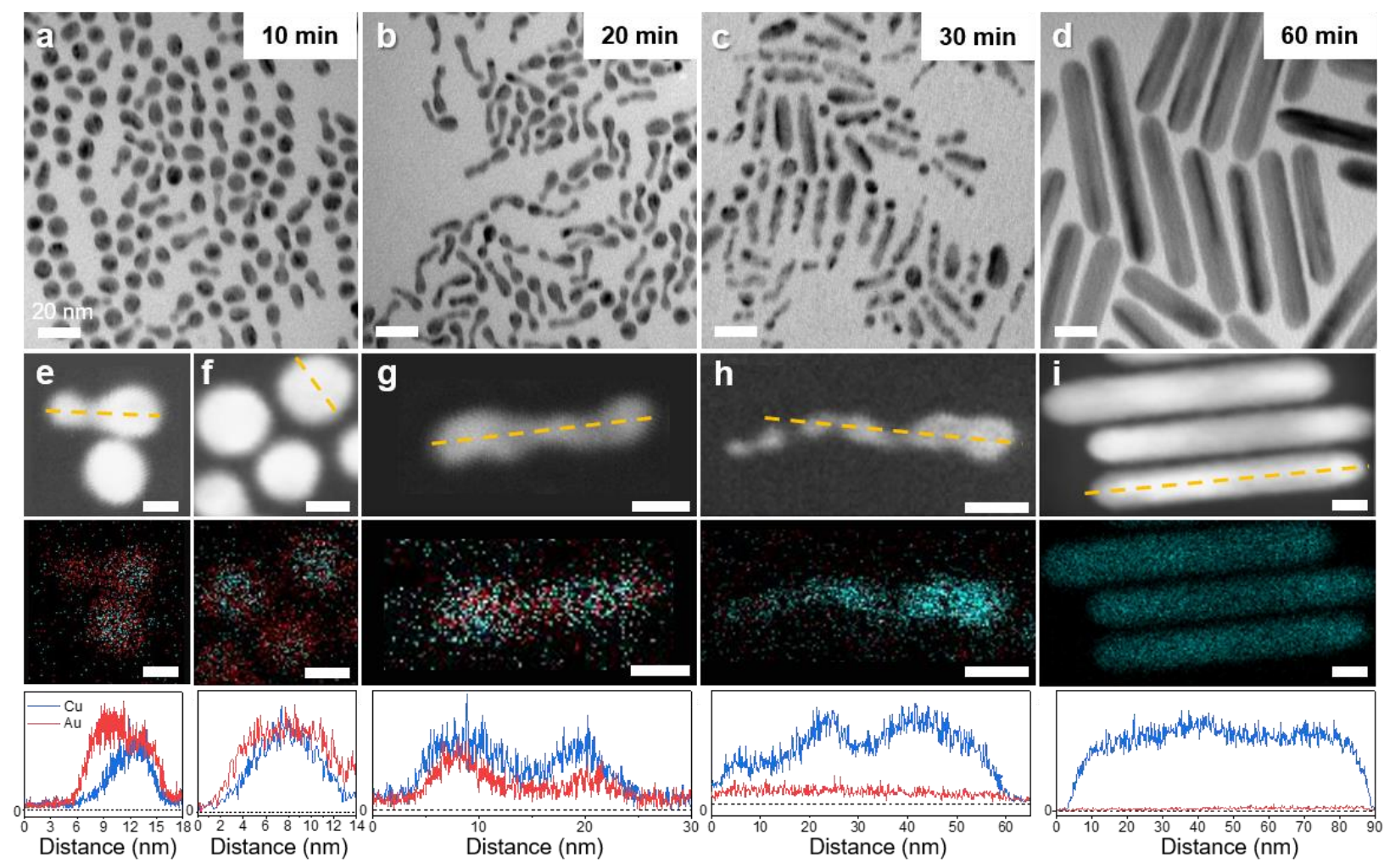

Figure S30. (a-d) TEM images and STEM-EDS analysis results of reaction intermediates of $\mathrm{Cu}$ NR synthesized by using $6.5 \mathrm{~nm}$ Au NCs as seeds. At $10 \mathrm{~min}$, a mixture of elongated (e) and spherical (f) NCs with a gold-rich surface was observed. At 20 min, zigzag-shaped NRs appeared $(\mathrm{b}, \mathrm{g})$. Between 20 and $30 \mathrm{~min}$, these NRs continued to grow via incorporation of more $\mathrm{Cu}$ atoms $(\mathrm{c}, \mathrm{h})$. Afterward, the surface of NRs became smoother as more $\mathrm{Cu}$ atoms were deposited, yielding highly uniform $\mathrm{Cu}$ NRs at $60 \mathrm{~min}(\mathrm{~d}$,i). STEM-EDS analysis revealed continuous enrichment of $\mathrm{Cu}$ concomitant with NR growth. Scale bars: $20 \mathrm{~nm}$ (a-d), $10 \mathrm{~nm}(\mathrm{e}-\mathrm{i})$. 


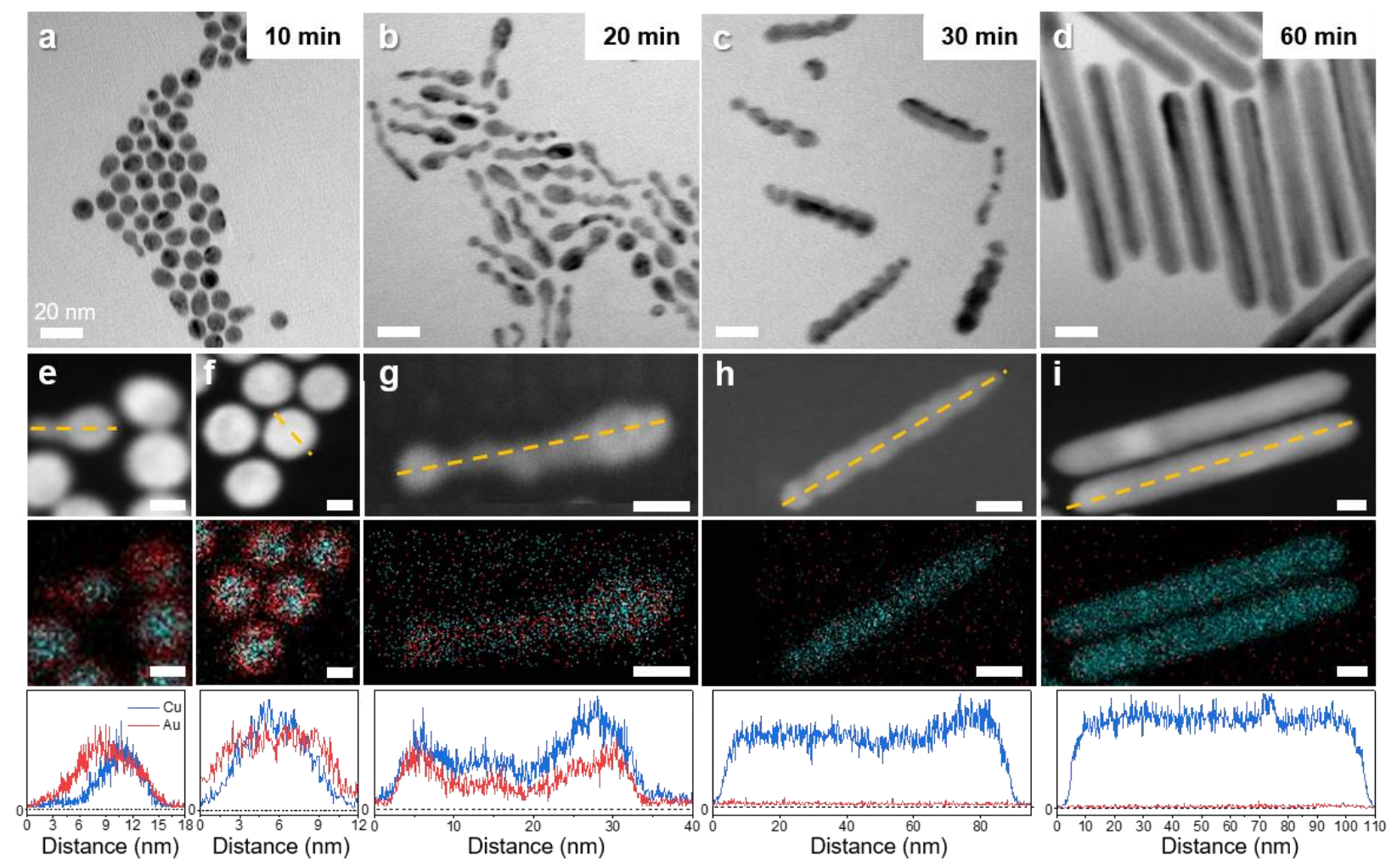

Figure S31. (a-d) TEM images and STEM-EDS analysis results of reaction intermediates of $\mathrm{Cu}$ NR synthesized by using $7.5 \mathrm{~nm} \mathrm{Au} \mathrm{NCs} \mathrm{as} \mathrm{seeds.} \mathrm{At} 10 \mathrm{~min}$, a mixture of elongated (e) and spherical (f) NCs with a gold-rich surface was observed. At 20 min, zigzag-shaped NRs appeared $(\mathrm{b}, \mathrm{g})$. Between 20 and $30 \mathrm{~min}$, these NRs continued to grow via incorporation of more $\mathrm{Cu}$ atoms $(\mathrm{c}, \mathrm{h})$. Afterward, the surface of NRs became smoother as more $\mathrm{Cu}$ atoms were deposited, yielding highly uniform $\mathrm{Cu}$ NRs at $60 \mathrm{~min}(\mathrm{~d}$,i). STEM-EDS analysis revealed continuous enrichment of $\mathrm{Cu}$ concomitant with NR growth. Scale bars: $20 \mathrm{~nm}(\mathrm{a}-\mathrm{d}), 10 \mathrm{~nm}(\mathrm{e}-\mathrm{i})$. 

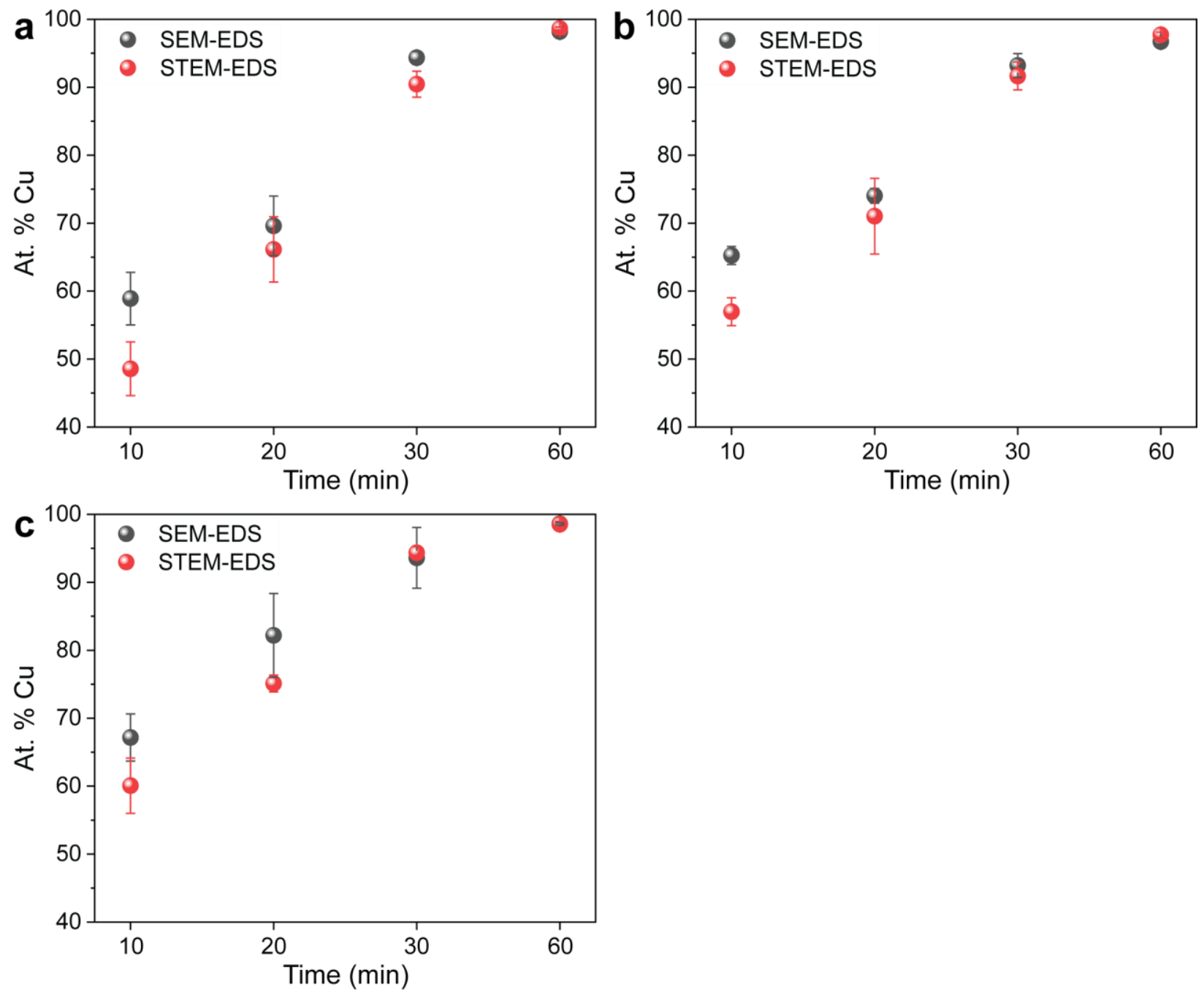

Figure S32. Time-dependent $\mathrm{Cu}$ contents determined from SEM-EDS (black dots) and STEMEDS (red dots) analyses of reaction intermediates of Cu NRs synthesized by using (a) $5.5 \mathrm{~nm}$, (b) $6.5 \mathrm{~nm}$, and (c) $7.5 \mathrm{~nm} \mathrm{Au} \mathrm{NC}$ seeds. 


\section{Supplementary References}

1. Zheng, N.; Fan, J.; Stucky, G. D. One-Step One-Phase Synthesis of Monodisperse NobleMetallic Nanoparticles and Their Colloidal Crystals. J. Am. Chem. Soc. 2006, 128, 65506551.

2. Wu, B.-H.; Yang, H.-Y.; Huang, H.-Q.; Chen, G.-X.; Zheng, N.-F. Solvent Effect on the Synthesis of Monodisperse Amine-Capped Au Nanoparticles. Chinese Chem. Lett. 2013, 24, 457-462.

3. Cui, F.; Yu, Y.; Dou, L.; Sun, J.; Yang, Q.; Schildknecht, C.; Schierle-Arndt, K.; Yang, P. Synthesis of Ultrathin Copper Nanowires Using Tris(trimethylsilyl)silane for HighPerformance and Low-Haze Transparent Conductors. Nano Lett. 2015, 15, 7610-7615.

4. Giuffrida, S.; Condorelli, G. G.; Costanzo, L. L.; Fragalà, I. L.; Ventimiglia, G.; Vecchio, G. Photochemical Mechanism of the Formation of Nanometer-Sized Copper by UV Irradiation of Ethanol Bis(2,4-pentandionato)copper(II) Solutions. Chem. Mater. 2004, 16, 1260-1266.

5. Jakubowski, W.; Kirci-Denizli, B.; Gil, R. R.; Matyjaszewski, K. Polystyrene with Improved Chain-End Functionality and Higher Molecular Weight by ARGET ATRP. Macromol. Chem. Phys. 2008, 209, 32-39.

6. Zhang, J.; Santos, P. J.; Gabrys, P. A.; Lee, S.; Liu, C.; Macfarlane, R. J. Self-Assembling Nanocomposite Tectons. J. Am. Chem. Soc. 2016, 138, 16228-16231.

7. Ye, X.; Jin, L.; Caglayan, H.; Chen, J.; Xing, G.; Zheng, C.; Doan-Nguyen, V.; Kang, Y.; Engheta, N.; Kagan, C. R.; Murray, C. B. Improved Size-Tunable Synthesis of Monodisperse Gold Nanorods through the Use of Aromatic Additives. ACS Nano 2012, 6, 2804-2817.

8. Chen, J.; Feng, J.; Yang, F.; Aleisa, R.; Zhang, Q.; Yin, Y. Space-Confined Seeded Growth of $\mathrm{Cu}$ Nanorods with Strong Surface Plasmon Resonance for Photothermal Actuation. Angew. Chem. Int. Ed. 2019, 58, 9275-9281.

9. Chen, L.; Ji, F.; Xu, Y.; He, L.; Mi, Y.; Bao, F.; Sun, B.; Zhang, X.; Zhang, Q. High-Yield Seedless Synthesis of Triangular Gold Nanoplates through Oxidative Etching. Nano Lett. 2014, 14, 7201-7206.

10. Babar, S.; Weaver, J. H. Optical Constants of Cu, Ag, and Au Revisited. Appl. Opt. 2015, $54,477-481$.

11. Liu, Z.; Zhong, Y.; Shafei, I.; Borman, R.; Jeong, S.; Chen, J.; Losovyj, Y.; Gao, X.; Li, N.; Du, Y.; Sarnello, E.; Li, T.; Su, D.; Ma, W.; Ye, X. Tuning Infrared Plasmon Resonances in Doped Metal-Oxide Nanocrystals through Cation-Exchange Reactions. Nat. Commun. 2019, 10, 1394.

12. Onsager, L. The Effects of Shape on the Interaction of Colloidal Particles. Ann. N.Y. Acad. Sci. 1949, 51, 627-659.

13. Liu, X.; Atwater, M.; Wang, J.; Huo, Q. Extinction Coefficient of Gold Nanoparticles with Different Sizes and Different Capping Ligands. Colloids Surf. B Biointerfaces 2007, 58, 37. 